Military Engineering

\title{
Patterned Non-affine Motion in Granular Media
}

John F. Peters and Laura E. Walizer

August 2012

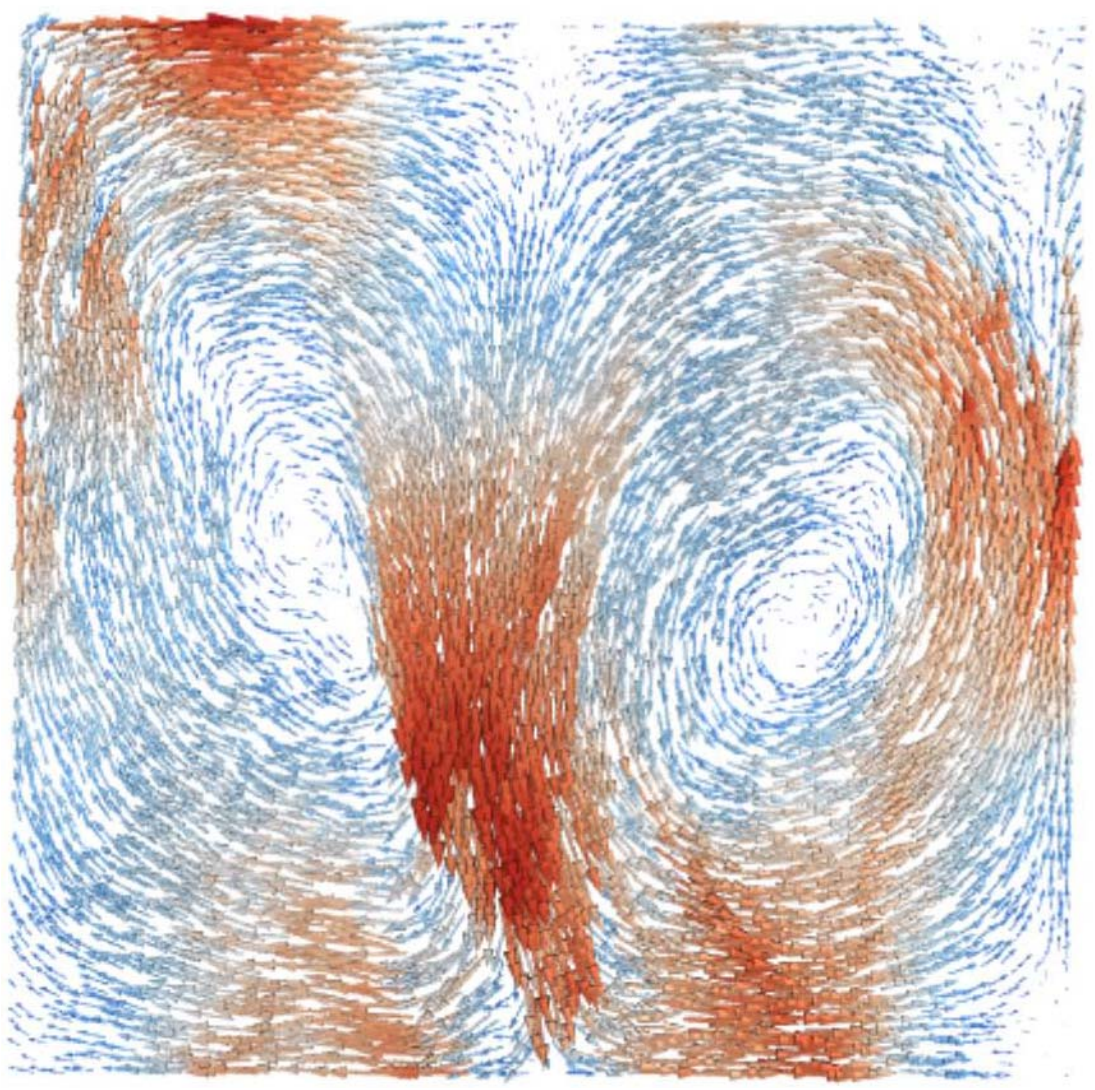




\title{
Patterned Non-affine Motion in Granular Media
}

\author{
John F. Peters and Laura E. Walizer \\ Geotechnical and Structures Laboratory \\ U.S. Army Engineer Research and Development Center \\ 3909 Halls Ferry Road \\ Vicksburg, MS 39180-6199
}

Final report

Approved for public release; distribution is unlimited.

\author{
Prepared for U.S. Army Corps of Engineers \\ Washington, DC 20314-1000 \\ Under U.S. Army Engineer Research and Development Center Military Engineering \\ Basic Research Program, Fundamental Investigation on Bridging \\ Between Microscale Phenomena and Macroscale Performance
}




\section{Abstract}

Vortex-like flow patterns often are observed in experiments on granular media for which uniform strain is expected based on the loading boundary conditions. These deformations become apparent when the motion associated with uniform strain is subtracted from the total particle motion. Besides presenting an interesting phenomenon that begs explanation, these vortex patterns suggest multi-scale structure to non-affine motion as suggested by modern continuum theories. Further, the rotational velocity field added to a uniform strain field produces a planar slip field. Thus, these structures are associated with the slip-band fields that eventually form and generally are associated with bifurcations in the solution path of the governing partial differential equations. A procedure is presented to extract these motion fields from discrete element simulations, along with conjugate forces associated with these motions. A key finding from the simulations is that the motions that eventually lead to shear band formation develop throughout the loading history rather than arising as a distinct bifurcation. Further, the pattern of rotational fields and, hence, the shear banding pattern are controlled by the boundary conditions. A question, only partly resolved here, is the origin of forces driving the rotational fields, considering no force exists at the boundaries in the case of frictionless platens.

DISCLAIMER: The contents of this report are not to be used for advertising, publication, or promotional purposes. Citation of trade names does not constitute an official endorsement or approval of the use of such commercial products. All product names and trademarks cited are the property of their respective owners. The findings of this report are not to be construed as an official Department of the Army position unless so designated by other authorized documents. 


\section{Contents}

Abstract...................................................................................................................................... if

Figures and Tables............................................................................................................ iv

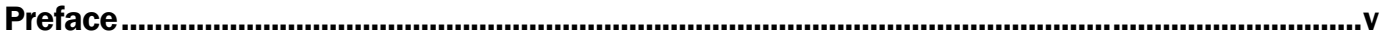

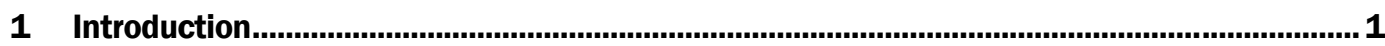

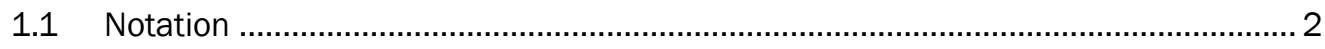

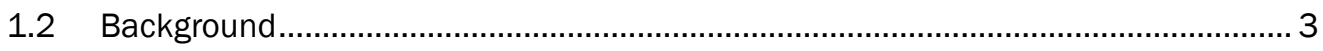

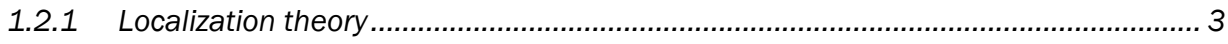

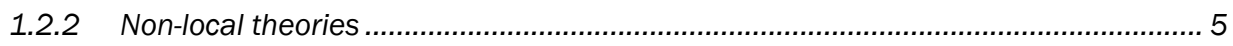

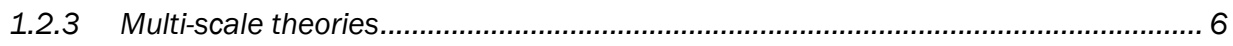

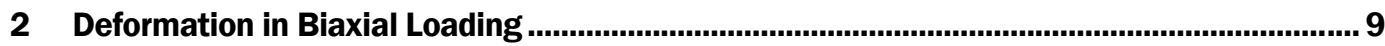

2.1 The simulation ................................................................................................ 10

2.2 General observations ............................................................................... 12

2.3 Analysis of deformation................................................................................ 14

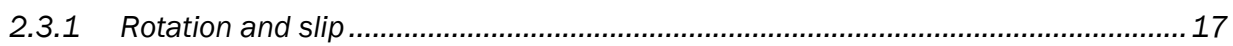

2.3.2 Particle rotations...................................................................................................... 18

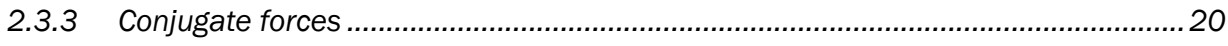

3 Results and Discussion..................................................................................................23

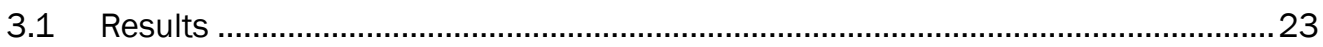

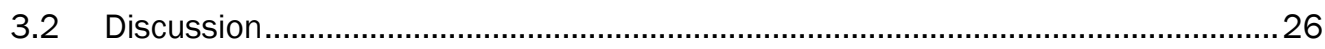

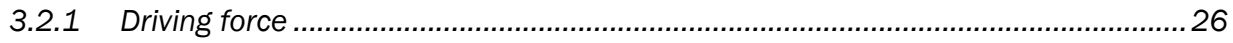

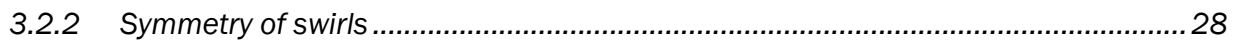

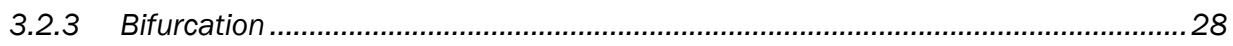

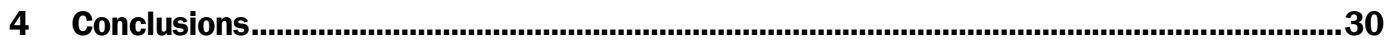

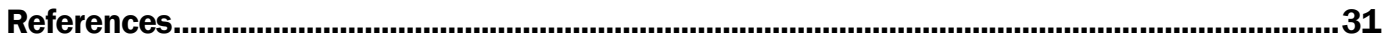

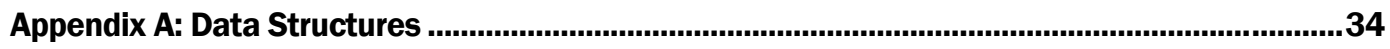

\section{Report Documentation Page}




\section{Figures and Tables}

\section{Figures}

Figure 1. Configuration of 9,409 particles and loading platens in a 2-D biaxial test. ....................... 9

Figure 2. Deformation patterns at final load step. ........................................................................... 11

Figure 3. Stress response to kinetic energy bursts......................................................................... 12

Figure 4. Kinetic energy displaying exponential decay when the system is allowed to relax............13

Figure 5. Development of non-affine motions in biaxial loading. The incremental movements (top) lack definite pattern, yet the accumulation of those motions (bottom) develop well-defined swirl pattern.

Figure 6. Coordinate system for biaxial simulation. Non-affine motion corresponds to case of friction on all four platens......

Figure 7. A slip field created from the addition of a uniform strain and a rotation.

Figure 8. Creation of a double shear banding pattern from addition of uniform strain to rotation field with mirror symmetry.

Figure 9. Components comprising the relative contact motion and contact force.

Figure 10. Relationship between strains computed for least-square fit of particle motions and total kinetic energy of particles.

Figure 11. Rate of swirl deformation based on least-square fit showing the early development of the swirl motion.

Figure 12. Development of force conjugate to the swirl deformation mode.

Figure 13. Particle rotations after full pattern development (left) and computed spin (right)

Figure 14. A scatterplot comparing actual and theoretically predicted displacements (left) and spins (right) for each particle in the swirl field.

\section{Tables}

Table 1. Parameters and properties used in the DEM simulation of a biaxial test 


\section{Preface}

The research described in this report was performed as part of a U.S. Army Engineer Research and Development Center (ERDC) Military Engineering Basic Research Program called the Fundamental Investigation on Bridging Between Microscale Phenomena and Macroscale Performance. The research was conducted by Dr. J ohn F. Peters, Geotechnical and Structures Laboratory (GSL), and Dr. Laura E. Walizer, Impact and Explosions Effects Branch (IEEB), Engineering Systems and Materials Devision (ESMD), GSL.

During this research effort, Henry S. McDevitt was Chief, IEEB;

Dr. Larry N. Lynch was Chief, ESMD; Dr. William P. Grogan was Deputy Director, GSL; and Dr. David W. Pittman was Director, GSL.

At the time of publication, Grogan was Acting Chief, GSL, and Dr. Gary Anderton was Acting Deputy Chief. COL Kevin J . Wilson was Commander and Executive Director of ERDC. Dr. J effery P. Holland was Director. 


\section{Introduction}

This report presents an analysis of sub-scale patterned deformation that is observed in granular media when large-scale deformation is subtracted. Examples of such observed patterns can be found in a variety of works (Williams and Rege 1997; Tordesillas et al. 2010; Koenders 1994; Gaspar and Koenders 2001; Kuhn 1999; and Rechenmacher 2006). One of the earliest observations in soil mechanics of such motion showed photographs of turbulent-like structures in sand under vibrational loading leading up to liquefaction (Mogami and Kubo 1953). Williams and Rege (1997) observed such structures in simulations using the Discrete Element Method (DEM) and used Proper Orthogonal Decompositions (PODs) to objectively capture the patterns. Kuhn (1999) and Kuhn (2003) also used DEM, and described several types of patterns associated with both displacement and rotations. Radjai and Roux (2002) described granular motions that were similar to those observed in fluids undergoing turbulence. Tordesillas et al. (2008) documented DEM simulations in which "vortex" patterns appear during monotonic loading as an ultimate "critical" state is obtained and noted the turbulent-like nature of the motion statistics associated with the patterns.

Such vortex patterns can be observed in the simple experimental case of a sample being loaded so that uniform strains are produced. In such a system, uniform deformations give way to spatial patterns of motion that possibly are associated with internal instabilities (Valanis 1996 and 1998). These instability-related deformation patterns are made apparent by subtracting deformations related to the uniform strain from the observed deformation, or otherwise applying a filter (Koenders 1994; Gaspar and Koenders 2001). Thus, the total deformation in these experiments is decomposed into uniform strain (affine deformation) and the sub-scale, patterned non-affine deformation. Rechenmacher (2006) made detailed observations of biaxial tests on sand that traced the evolution of shear bands. Within the bands, non-affine motions often take on rotational patterns. It is not clear that nonaffine motions are necessarily rotational, although that appears to be a common observation in the types of experiments that will be discussed in this report. For purposes of discussion, these motions will be referred to as swirls. 
The fact that these sub-scale deformations emerge as well-organized patterns is worthy of study in itself. Here, we argue that the existence of such patterns suggests a multi-scale approach to modeling granular media. Further, these deformations bring into question the fundamental assumption of localization (shear banding) theory (i.e. that localized bands of shear emerge as bifurcations in the solution to the boundary value problems as a result of instabilities in the constitutive response of the material). Such patterns evoke interest not only because their existence is unexplained, but also because describing their role in the mechanics of granular media can provide links to richer continuum theories based on micromechanical and multi-scale concepts.

The physical significance of the non-affine swirl motion is that it violates the definition of continuum deformation. Motion in a continuum is such that the relationship between each material point and its local neighbors remains constant from a topological perspective. Thus, if a material point lies within a cage of material points prior to deformation, it will remain within the cage after the deformation. The fact that discrete particles of finite size are considered suggests that such violations of continuum motions are likely. Swirl patterns imply a relative motion in which particles can migrate from their cages diffusively. This fact suggests that multi-field theories are necessary to describe these systems, such as the theories proposed by Valanis (1996) or those currently applied in multi-scale models (Kadowaki and Liu 2004; Kadowaki and Liu 2005), which are generalizations to the multi-scale concepts originally proposed by Germain (1973). The relationship of these multi-field formulations to localization theory comes from the desire to avoid mathematical difficulties associated with solving boundary value problems that arise using constitutive equations based on the local continuum formulation (Valanis and Peters 1996)

In this report, patterned deformations are addressed in the limited context of an academic simulation of a single, ideal two-dimensional (2-D) biaxial experiment. However, considering the observation of such features in physical experiments in sand by Rechenmacher (2006), the procedures described have wider application.

\subsection{Notation}

Two forms of notation are used in this report. Conventional tensorial notation, in which repeated indices indicate summation, is used for expressing fundamental relationships applied to quantities associated with 
a continuum point or a particle. A comma between indices indicates differentiation with respect to the second index. Matrix notation is used to express these relationships when dealing with particle statistics. The relationship between vector and tensor quantities and their matrix counterparts is discussed in Appendix A.

\subsection{Background}

The introductory discussion begins with localization theory, non-local theory, and multi-scale analysis, with a view toward demonstrating their interrelationship. This interrelationship is the basis of the analysis of the non-affine deformation.

\subsubsection{Localization theory}

Intensive research over the past five decades has led to the conclusion that local continuum theories are not adequate to formulate initial-boundary value problems for frictional materials (Valanis and Peters 1996). The central problem is that plastic deformation in frictional materials is described through a non-associated flow rule, which imparts non-positive characteristics to the response similar to strain softening and results in mathematically ill-posed problems. The mathematical difficulty associated with such materials prompted many attempts to develop enriched continuum theories that capture important instabilities yet are well posed. Such theories include those that incorporate higher strain gradients in their constitutive relationships (Vardoulakis and Aifantis 1991), multi-field theories, e.g. the Cosserat Theory (Sluys 1992; De Borst and Sluys 1991), and non-local theories that express constitutive relationships in the form of integral equations. These theories collectively will be referred to as nonlocal theories.

The focus of many investigations is the condition under which localized shear bands appear in the course of loading to failure. As a practical matter, localization bands are commonly observed phenomena that dominate soil behavior in the limit state. The principal mathematical theory for capturing such bands has centered on the notion that localization represents a bifurcation in the solution to the boundary-value problem or, equivalently, loss of ellipticity in the spatial operators of the initial-boundary value problems (Rice 1977). Unfortunately, the same hypothesis virtually invalidates the use of constitutive models that permit localization because, with the loss of ellipticity comes ill-posedness, which in the case of numerical solutions is 
expressed as sensitivity to mesh details and inability to capture shear band thickness. The latter problem is particularly severe because it leads to an infinite softening upon mesh refinement (Bazant and Belytschko 1985) that in turn leads to a situation where it is impossible to simulate the experiments from which the material parameters were derived. Valanis and Peters (1996) noted that materials following an associated flow rule also reach a state where ellipticity is lost, which is consistent with reaching a failure state and therefore physically normal. On the other hand, the non-associated flow law permits failure states that are pathological because they are not associated with the limiting condition of the material. Valanis and Peters also note that many of these pathologies are observed in experiments and that shear banding theories give a "very good qualitative description of shear banding." The distinction between the failure condition, in which the material loses its capacity to resist homogeneous shear stress, and the shear localization condition, in which resistance is lost along a distinct direction, was made by Rice (1977) and given a complete analysis by Rudnicki and Rice (1975). Their results are supported by the outcomes of experiments. The potential for shear banding to appear in experiments at stress states well below the failure surface is described by Peters et al. (1986) whereby, as predicted by Rudnicki and Rice (1975), the cylindrical triaxial test specimens were highly resistant to shear banding, while specimens under plane strain conditions unmistakably failed by shear banding prior to reaching the ultimate failure condition. Other behavioral oddities of frictional materials predicted by theories based on non-associated flow laws have been confirmed by experiment (Lade et al. 1993).

Real experimental verification of shear banding theory only can come when the formation of shear bands can be predicted in numerical simulations, free from mesh dependency and with proper convergence of the solution. Merely predicting a state in which shear bands can appear is not sufficient from a scientific perspective and certainly inadequate for engineering analyses of earthen structures. In fact, shear banding theory does not predict localization. Rather, the theory predicts the existence of directions along which the material does not resist traction (Rice 1977). In such a state, localization is only one of many potential deformation patterns that satisfy both governing equations and boundary conditions for a particular problem. It appears, therefore, that although plasticity models based on a nonassociated flow law potentially can predict the wide range of instabilities observed experimentally, the application of those theories necessitates 
abandoning local continuum theory (Valanis and Peters 1996), which begs the question of how extended theories should be developed.

\subsubsection{Non-local theories}

Extended continuum theories date at least from the Cosserat brothers (Cosserat and Cosserat 1909) and have been a rich area of research in continuum mechanics since the 1950s (McDowell 2000), with particular influence by Mindlin (1964). The application of these theories to full-scale numerical simulations is a development of the past two decades. Sluys (1992), and De Borst and Sluys (1991) both investigated non-local continuum laws that would regularize problems formulated from constitutive laws with strain softening and non-associativity. In this context, regularizetion is the mathematically motivated addition to a problem formulation to somehow obviate the ill-posed character of the model. However, there are physical motivations for extending continuum theory to include non-local effects. Mühlhaus and Vardoulakis (1987) made considerable progress with Cosserat theory, predicting occurrence, orientation, and thickness of shear bands. A rate equation based on Cosserat theory similarly was employed by Tordesillas et al. (2004) to derive solutions for shear localization that allowed the evolution of the band to be followed beyond its initial formation. Notable in this regard was the finding that softening within the band is required for localization to occur (Bardet and Proubet 1992). Walsh and Tordesillas (2006) employed Cosserat models in finite element analyses that included full development of failure mechanisms without incurring numerical pathologies found in local continuum formulations. The work of Tordesillas et al. (2004) was centered on micromechanical considerations, thus the motivation for the work went beyond the regularization issue. In particular, later work by Tordesillas et al. (2010) investigated the relationship between localization and meso-scale features such as force chains. Another trend in recent years is multi-scale analysis using hierarchical continuum theories, as employed by Kadowaki and Liu (2004 and 2005) to both regularize the boundary value problem and to provide a framework for incorporating information from microscale information.

Experimental data to support these theories came from research in shear localization in which careful measurements of thickness, particle kinematics, and evolution provided data for comparison to analytical results but to a much lesser extent information for development of continuum theories. Unfortunately, available experimentation only provides half the picture. To make advances in non-local continuum 
theory, information is required on both kinematics and kinetics in a multiscale setting. Advances in tomography and x-ray analysis provide some optimism, although the precision of the data required to describe evolution of grain motion and inter-grain forces is years away. Photoelastic methods developed by Behringer and his coworkers (Utter and Behringer 2009) is at present the only experimental method to provide both particle motion and details on inter-particle forces.

Numerical simulations using the DEM (Cundall and Strack 1979) in recent years achieved considerable popularity for investigating granular media. Like the photo-elastic method, both displacements and forces can be obtained. In addition, with the method comes the equations that govern the behavior at the particle scale. Unfortunately, much of what we see in granular media is emergent behavior that only can be obtained by integrating DEM equations and do not give direct insight by themselves. Thus, one of the hopes driving micromechanical modeling (i.e. to synthesize the constitutive behavior of granular media using the particle-scale laws alone) appears to be fundamentally out of reach. What the DEM can provide is the virtual laboratory to create higher-level continuum models using data from particle-scale measurements rather than through bulk-scale experiments. Such a goal is within reach, given the wealth of development in continuum theory.

\subsubsection{Multi-scale theories}

Multi-scale formulations define a continuum in which multiple fields interact incompatibly, each representing a particular process or scale. The Cosserat theory is an example in which a field is added consisting of rotational degrees of freedom, and their conjugate couples, that are independent from the spin of the continuum in which they are embedded, thus capturing some aspects of discrete granular media not possible with conventional continuum theory. Germain (1973) introduced the multi-field concept to capture micro-structure based on virtural power arguments. Germain's formulation was generalized by Liu and his co-workers (Liu et al. 2006) as a multi-resolution representation where each field represents a length scale driven by the constitutive response typical of that scale.

Specifically, Valanis, who in a series of papers (1996, 1998, and 2001) investigated the mathematical structure required for continuum theories to capture patterned deformations, presents a multi-field theory based on a variational formulation that introduces multiple fields as internal 
variables. As for traditional continuum, the equilibrium is written in terms of the average Cauchy stress and a general body force, $f_{i}$,

$$
\frac{\partial \bar{\sigma}_{i j}}{\partial x_{j}}=f_{i}
$$

noting that the stress is related to the free energy and the strain through $\bar{\sigma}_{i j}=\partial \not / \partial \epsilon_{i j}$, where $\epsilon_{i j}$ is the symmetric part of the gradients $\bar{u}_{i, j}$. In addition to the average deformation $\bar{u}_{i}$ with its gradient $\bar{u}_{i, j}$ and respective conjugate forces $f_{i}$ and $\bar{\sigma}_{i j}$, Valanis (1996) posits internal motions $q_{i}$ with respective forces $Q_{i}$. The feature that distinguishes this theory from previous formulations based on internal variables is the inclusion of the gradient $q_{i, j}$ in the free energy, which accordingly introduces the internal force $Q_{i j}$ and produces a field equation

$$
\frac{\partial \psi / \partial q_{i, j}}{\partial x_{j}}+\frac{\partial \psi}{\partial q_{i}}=Q_{i}
$$

where the free energy is given by $\psi=\psi\left(u_{i, j}, q_{i}, q_{i, j}\right)$ and $Q_{i j}=\partial \psi / \partial q_{i, j}$.

Valanis' formulation is based on the concept that $q_{i}$ represents sub-scale motions that are orthogonal to the average continuum motions in that on any surface $S$

$$
\int_{s} u_{i} q_{i} d S=0
$$

Similarly,

$$
\int_{s} T_{i} Q_{i} d S=0
$$

where $T_{i}$ is the surface tractions equilibrating $\bar{\sigma}_{i j}$. Subsequent sections of this report will show, as have others, it always is possible to decompose motions into average and variable parts such that the two fields are orthogonal. Multi-scale theories make the critical connection to the idea that these motions are associated with forces, independently contribute to the free energy, and thus can be represented as a separate field. Moreover, by introducing the gradients of these motions into the free energy, the field 
equations must satisfy boundary conditions, which affords new opportunities for capturing effects seen in experiments but not predicted by traditional continuum theory. In the case of the Valanis formulation (1996) on boundaries, $\partial u$, where displacement is specified

$$
q_{i} n_{i}^{\partial u}=0
$$

and on boundaries $\partial T$, where traction is specified

$$
Q_{i j} n_{j}{ }^{\partial T}=0,
$$

where, $n_{i}^{b}$ is the unit vector normal to boundary $b$. 


\section{Deformation in Biaxial Loading}

The research presented here is built around the biaxial loading test that is commonly the focus of granular media studies using both photo-elastic and DEM methods. The biaxial test consists of a rectangular domain of particles compressed between two sets of platens, as shown in Figure 1. (The platen configuration shown corresponds to that required in an actual experiment. In the simulations, the platens do not interact, making the complex geometrical arrangement unnecessary.) Loading consists of two phases: initialization, during which the particles are placed within the domain and loaded isotropically by applying forces to the plattens, and shearing, during which the platens are displaced under constant rates such that the volume of the specimen remains constant. The platens are assumed to be frictionless such that forces between platens and particles are purely normal to the contact plane. No gravity forces were assumed for initial loading or for subsequent shearing.

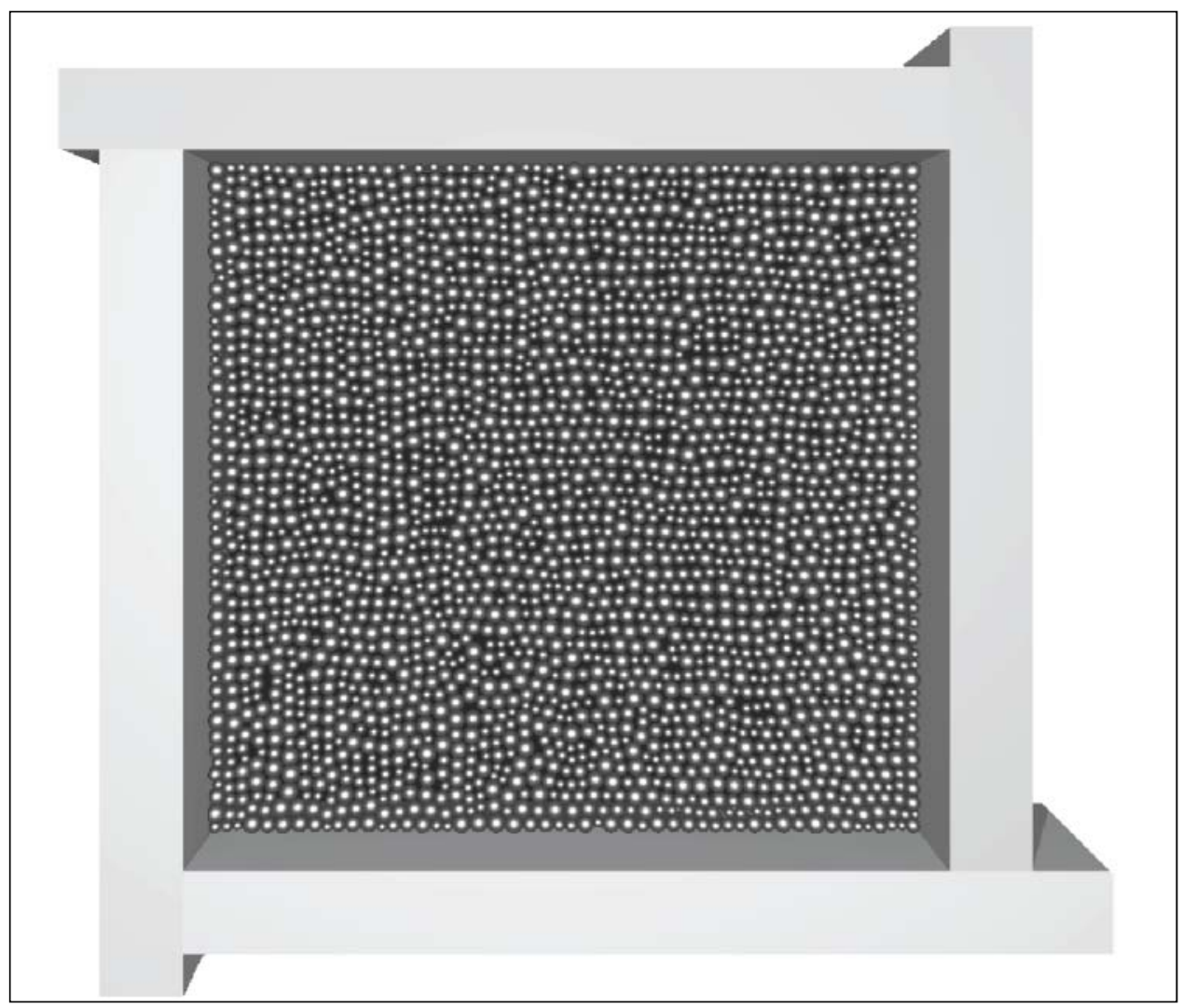

Figure 1. Configuration of 9,409 particles and loading platens in a 2-D biaxial test. 


\subsection{The simulation}

The simulations were performed using a three-dimensional (3-D) discrete element modeling code originally described by Horner et al. (2001). A 2-D domain was achieved by aligning all particle centers to lie in a plane and applying only loads that lie within the plane. All particles had perfectly spherical shapes. Therefore, the particles made contact only at their symmetry planes, and this configuration thus corresponds to a 2-D array of discs. This arrangement remains stable without lateral support because, provided the particle center coordinates lie in the same plane to within machine precision, there are no out-of-plane forces.

Although circular discs or spheres have been used extensively in discrete element simulations, they have the drawback of susceptibility to excessive instabilities by particle rolling. To limit destabilizing particle rotation, a small rolling resistance can be applied at the contacts similar to that employed by Tordesillas and Walsh (2002). The limiting moment is computed through a frictional rolling resistance law.

Contact damping of normal motion was achieved through a hysteretic law in which the stiffness for unloading the contact is greater than that for loading. The load-unload stiffness ratio was selected to give a nominal coefficient of restitution of 0.1. Damping in the shear response was the result of frictional sliding. Within unload-reload cycles, linear viscous damping was added to the contact response to eliminate high-frequency oscillations.

Contact properties were selected to limit inter-particle penetration while yielding a reasonable computational time step. The average interpenetration at particle contacts was $1.2 \%$ of the particle radius. The properties are shown in Table 1.

The particle locations were initialized through a growth procedure in which seed particles are placed randomly in a regular grid of cells within the domain, then grown to their final size in increments as the platens are allowed to compress the particle mass (Matous et al. 2007). The initial placement of the seed particles is based on estimating the number of particles needed to fill the desired space given their final target size. The final sizes of the particles were selected randomly from a uniform distribution in which the largest particle is twice the size of the smallest particle. The initial porosity, computed as area of void space divided by total area 
Table 1. Parameters and properties used in the DEM simulation of a biaxial test.

\begin{tabular}{|l|l|l|}
\hline Property & Units & Value \\
\hline Max Radius & $\mathrm{m}$ & 0.250 \\
\hline Min Radius & $\mathrm{m}$ & 0.125 \\
\hline Normal Stiffness & $\mathrm{kN} / \mathrm{m}$ & 5000 \\
\hline Shear Stiffness & $\mathrm{kN} / \mathrm{m}$ & 1000 \\
\hline Coefficient of restitution & - & 0.1 \\
\hline Contact Friction (sliding) & - & 0.5 \\
\hline Contact Friction (rolling) & - & 0.5 \\
\hline Number of Particles & - & 9409 \\
\hline Initial Specimen Height & $\mathrm{m}$ & 35.533 \\
\hline Initial Specimen Width & $\mathrm{m}$ & 35.516 \\
\hline Initial Area Porosity & - & 0.15 \\
\hline
\end{tabular}

within the platens, was 0.15 . As a reference, the area porosity of equal-size discs in square and hexagonal arrangements is 0.21 and 0.093 , respecttively. Because the particles are compressed as they grow, a greater degree of compaction than would be expected can be achieved with compression from a loose state alone.

Vector plots of particle displacements between the initial and final configurations are shown in Figure 2. Deformations near the end of loading have localized into a set of $\mathrm{V}$-shaped shear zones shown in Figure 2a. On average, the shear localization zone width corresponded to 10 particle diameters. The deformation for a homogeneous specimen should correspond to a uniform straining, with the associated velocities shown in Figure 2b. The difference between the computed uniform straining deformation and the actual deformation is the double swirl pattern shown in Figure 2c.

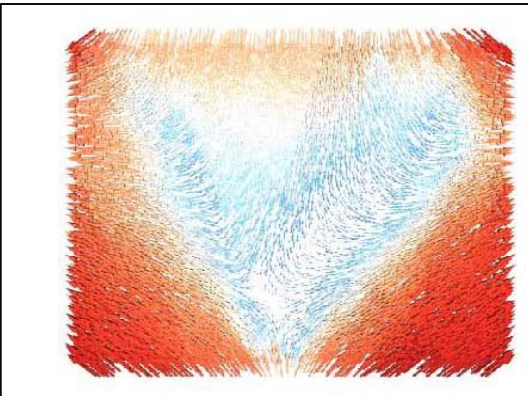

a) Final Total Deformation

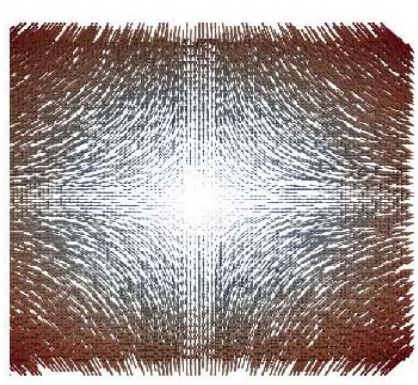

b) Affine Deformation

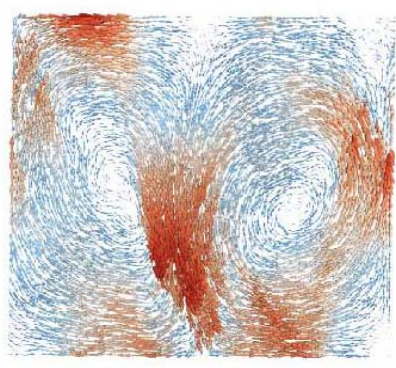

c) Final Deformation with Affine Motion Removed

Figure 2. Deformation patterns at final load step. 


\subsection{General observations}

As reported by Tordesillas et al. (2004), deformation is neither uniform in space nor continuous in time and is punctuated with bursts of kinetic energy. Non-affine deformation tends to be associated with bursts in kinetic energy that occur at various times throughout the specimen. These bursts are followed by abrupt reductions in shear stress, as shown in Figure 3. After each burst, there is a period where these motions are damped and the kinetic energy reduces to that associated with the affine motion of the particle field, during which time the stress builds to the average trend it followed prior to the drop. An example of this damping is illustrated by stopping the load increase and allowing the particle system to come to a rest state at a constant load. During this rest period, the kinetic energy appears to undergo an exponential decay, as shown in Figure 4, suggesting that the energy that is damped through inter-particle collisions decays at a rate proportional to the kinetic energy.

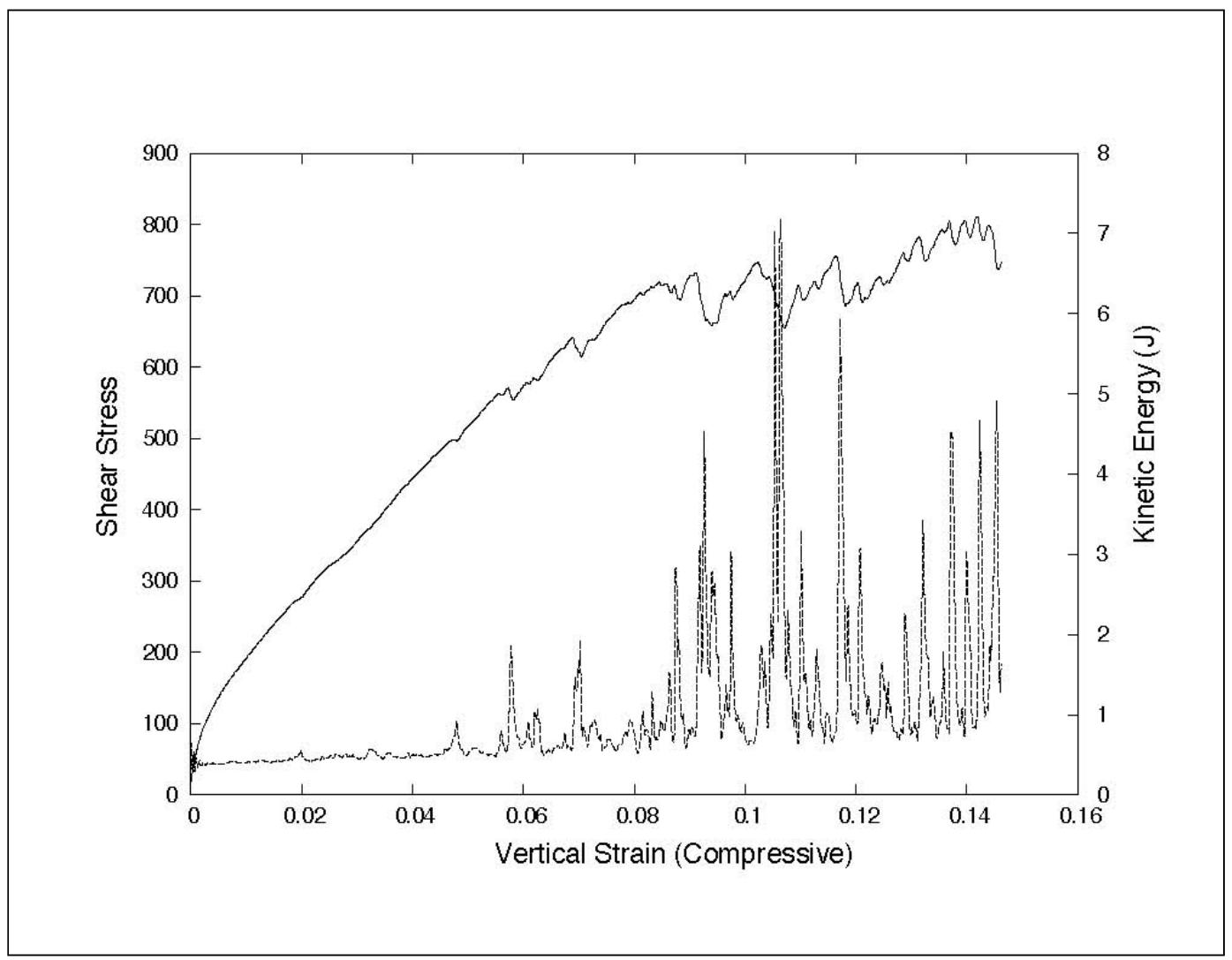

Figure 3. Stress response to kinetic energy bursts. 


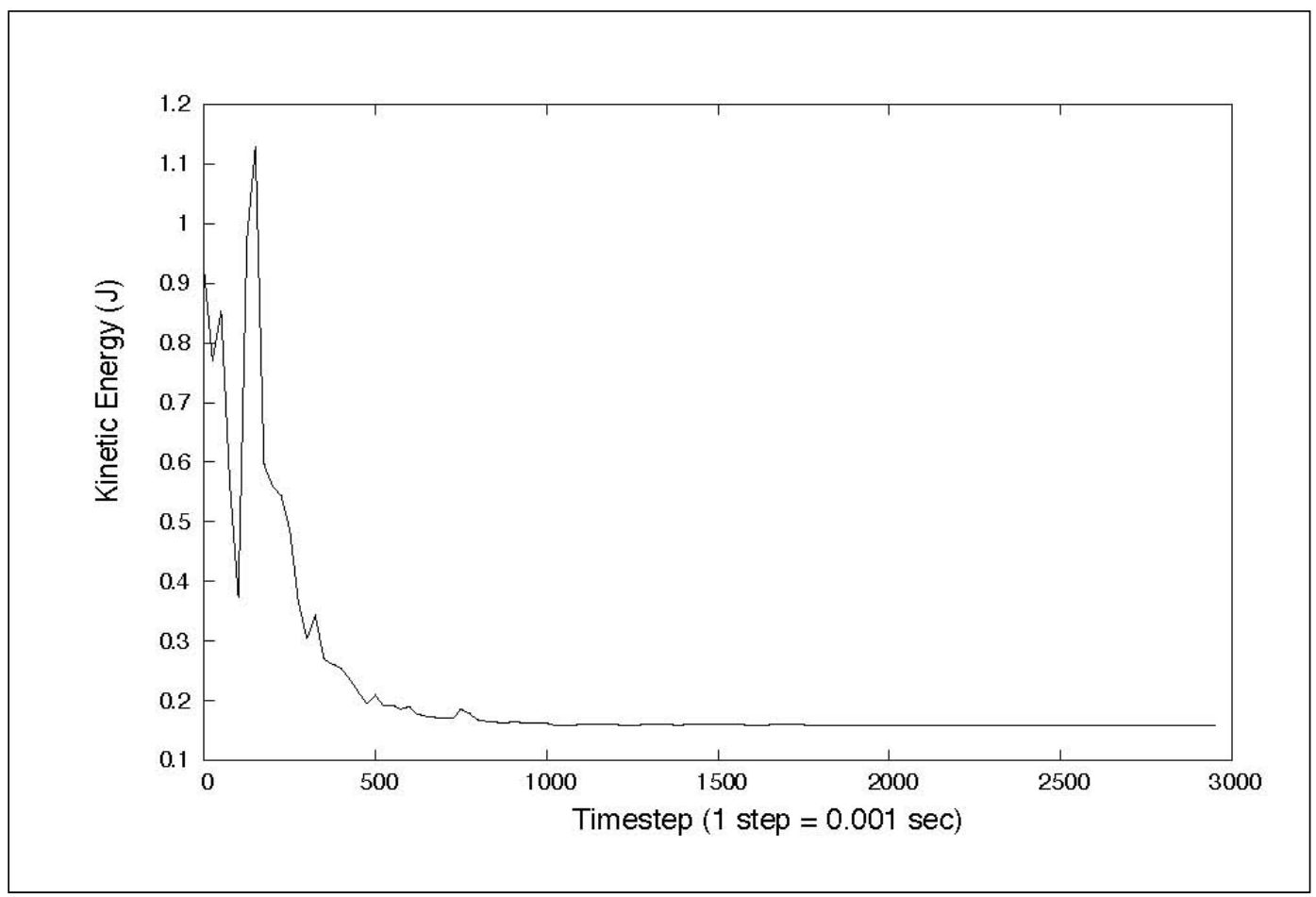

Figure 4. Kinetic energy displaying exponential decay when the system is allowed to relax.

The kinetic energy bursts are episodic deviations from affine deformation that seem to occur in random locations. However, the cumulative pattern of non-affine deformation clearly has a structure that is revealed by plotting the total non-affine deformation for each particle $v^{p}(t)$ consisting of the difference between initial locations and the locations at $t$ (Figure 5). Within the sequence of increments shown, the swirl shapes are perceptible in the first increment, obvious by the second increment, and intensify to dominate the non-affine motions in the remaining increments.

A close inspection of Figure 2 shows the velocity patterns to be parallel to the boundaries. These patterns are obtained by subtracting the affine deformation from the total deformation. The affine deformation is computed from the movement of the walls. At the platens, the particles touching the walls move with the affine deformation in the direction of the wall. The non-affine deformation, therefore, must be parallel to the wall by definition. Away from the wall, the non-affine motion forms two rotational cells, with the left rotating in the clockwise direction and the right rotating in the counterclockwise direction, creating mirror symmetry about the centerline of the specimen. Comparing the non-affine motion to the total deformation in Figure 2 reveals that the ultimate location of the localized shear zones coincides with the locations of the evolving swirls. 


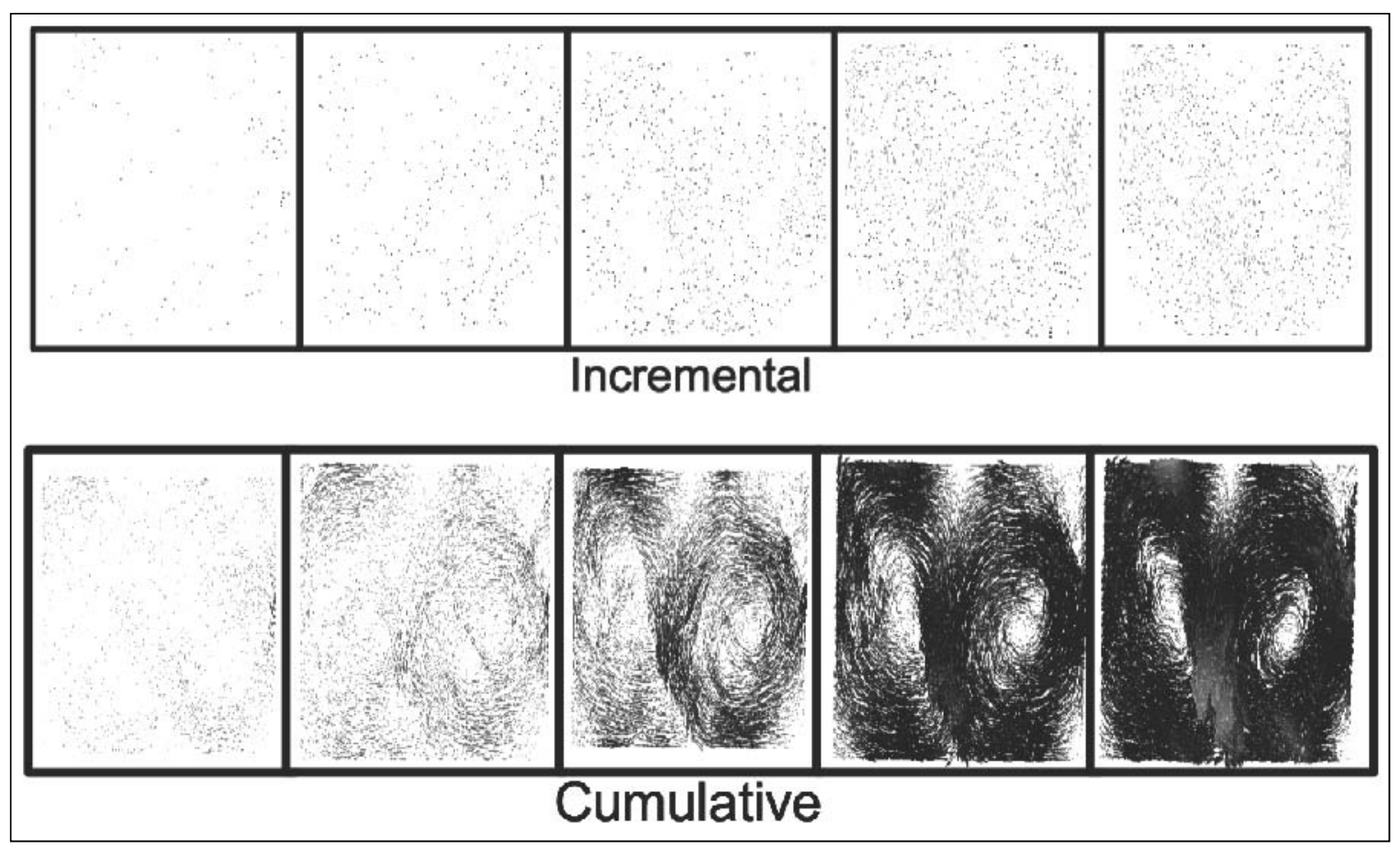

Figure 5. Development of non-affine motions in biaxial loading. The incremental movements (top) lack definite pattern, yet the accumulation of those motions (bottom) develop well-defined swirl pattern.

Although the analysis concentrates on frictionless walls, the effect of adding friction to the walls is notable for both the relationship between slip and swirl patterns and the effect of boundary conditions on the patterns that emerge. The frictionless case is important as a matter of principle because, in the absence of platen-particle friction, the affine deformation should be homogeneous, leading to a clearer distinction between affine and non-affine deformation. In addition, because boundary tractions are zero in the directions of the non-affine motion, a clearer case can be made that coupling must exist between the affine and non-affine fields. When friction is added, swirl patterns emerge but are affected by the boundary tractions that develop. Figure 6 shows the swirl patterns developed for the case in which all platens are frictional, whereby four swirl patterns emerge coinciding with double shear bands that meet in the specimen center. For the case where one platen has friction, two swirl patterns emerge, coinciding with double shear bands that meet on the boundary of the specimen.

\subsection{Analysis of deformation}

The analysis of deformation initially is built around extracting the uniform part of the motion, which is interesting because the ideal strain can be computed from motions of the boundaries and thus serves as a reference. 


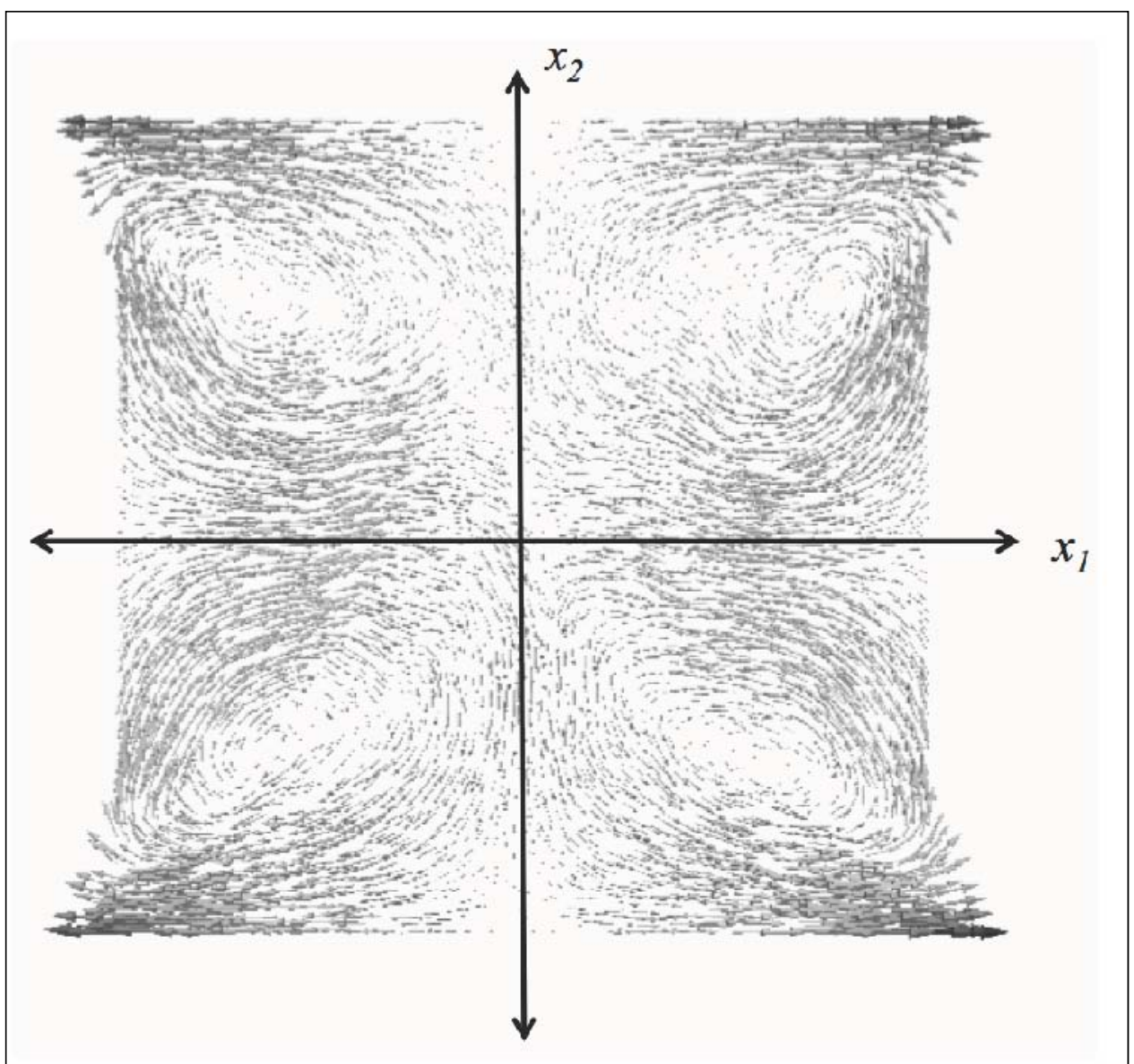

Figure 6. Coordinate system for biaxial simulation. Non-affine motion corresponds to case of friction on all four platens.

For a coordinate system centered at the specimen centroid, the motion, $\bar{u}_{i}$ of a particle at location $x_{i}$, corresponds to uniform strain $\epsilon_{i j}$ when $\bar{u}_{i}=\epsilon_{i j} x_{j}$. The non-affine motion is given by the difference between the observed motion and the affine motion, i.e.

$$
\begin{aligned}
v_{i}^{\prime} & =u_{i}-\bar{u}_{i} \\
& =u_{i}-\epsilon_{i j} x_{j}
\end{aligned}
$$

For purposes of the analysis to follow, consider vector $u$ and $v^{\prime}$ composed of the respective velocity components for each particle and arranged in vectors, as described in Appendix A. Similarly, matrix $L$ and vector $e$ are defined from the particle coordinates and the components of $\epsilon_{i j}$ to obtain an equivalent relationship to Equation 8:

$$
v^{\prime}=u-L e
$$


The vector $e$ contains, in addition to the strains, the rigid-body velocity and is necessary to properly represent general deformations. Accordingly, $L$ contains the necessary terms to project those components onto the velocity field. A least-square approximation to $\epsilon_{i j}$ is obtained by minimizing $R^{2}=v^{\prime T} v^{\prime}$, to get

$$
e=\left(L^{T} L\right)^{-1} L^{T} u
$$

Note that the least-square approximation of $e$ (discussed later in detail and shown in Figure 10) differs from the uniform strain computed from the platen motion, as was demonstrated by Bagi (2006). The lack of fit can be anticipated from considering a one-dimensional deformation. The best-fit line through a plot of displacement versus distance would not, in general, pass through the displacement values at the boundary points used to compute the real strain.

An intermediate result in minimizing $R^{2}$ is $L^{T}(u-L e)=O$ and implies that $\bar{u}_{i}$ and $v_{i}^{\prime}$ are orthogonal, leading to the important relationship

$$
L^{T} v^{\prime}=0,
$$

In addition, because $\bar{u}_{i}$ was constructed to contain rigid-body motion, $v^{\prime}$ represents a deformation component of the particle mass (Peters 2005).

Next, consider the deformation as the linear combination of two orthogonal patterns $u_{i}=\bar{u}_{i}+v_{i}^{\prime}$; for the biaxial test, $\bar{u}_{i}=\epsilon_{i j} x_{j}$. The nonaffine motion is the product of a pattern qi and a magnitude $\alpha$. The magnitude of $q_{i}$ is arbitrary because it defines only the pattern of motion. Following the previously defined convention of expressing the relationship in the matrix notation, the displacement is given by

$$
u=L e+\alpha q,
$$

where $L^{T} q=0$ and $q^{T} q=1$. It follows that, by pre-multiplying Equation 11 by $L^{T}$, one readily obtains the least-square expression for $e$. Similarly, by pre-multiplying Equation 11 by $q^{T}$, the magnitude of the non-affine motion is obtained

$$
\alpha=q^{T} u .
$$


The decomposition of motions can be extended to additional terms representing the deformation as a linear combination of mutually orthogonal unit direction vectors that span the space of possible motions. The magnitude for each term is found as the projection of the total displacement on the direction vector.

\subsubsection{Rotation and slip}

The relationship between the swirl patterns and localized shear band is no coincidence, as can be illustrated with a simple analysis. For a coordinate system with the origin at the center of the specimen, the velocities derived from a constant strain field are given as

$$
\begin{aligned}
& \bar{v}_{1}=\epsilon_{1} x_{1} \\
& \bar{v}_{2}=\epsilon_{2} x_{2} .
\end{aligned}
$$

In the case of constant-volume motion, $\epsilon_{1}=-\epsilon_{2}=\varepsilon$, a rotational field is created by switching the coordinates as follows

$$
\begin{aligned}
& v_{1}^{\prime}=\varepsilon x_{2} \\
& v_{2}^{\prime}=-\varepsilon x_{1} .
\end{aligned}
$$

Adding these two fields for constant volume flow gives the total deformation as

$$
\begin{aligned}
& v_{1}=\varepsilon\left(x_{2}-x_{1}\right) \\
& v_{2}=-\varepsilon\left(x_{2}-x_{1}\right)
\end{aligned} .
$$

Notice that the total flow is such that $v_{1} / v_{2}$ is constant, giving rise to the planar flow pattern illustrated in Figure 7.

Swirl patterns that satisfy the kinematic boundary conditions for the nonaffine motions in a rectangular domain having dimensions $2 L_{1} \times 2 L_{2}$ can be expressed in trigonometric form as

$$
\begin{aligned}
& v_{1}^{\prime}=\alpha_{1}(m, n) \sin \left(\frac{m \pi x_{1}}{2 L_{1}}+\frac{m \pi}{2}\right) \cos \left(\frac{n \pi x_{2}}{2 L_{2}}+\frac{n \pi}{2}\right) \\
& v_{2}^{\prime}=\alpha_{2}(m, n) \cos \left(\frac{m \pi x_{1}}{2 L_{1}}+\frac{m \pi}{2}\right) \sin \left(\frac{n \pi 2 x_{2}}{2 L_{2}}+\frac{n \pi}{2}\right),
\end{aligned}
$$




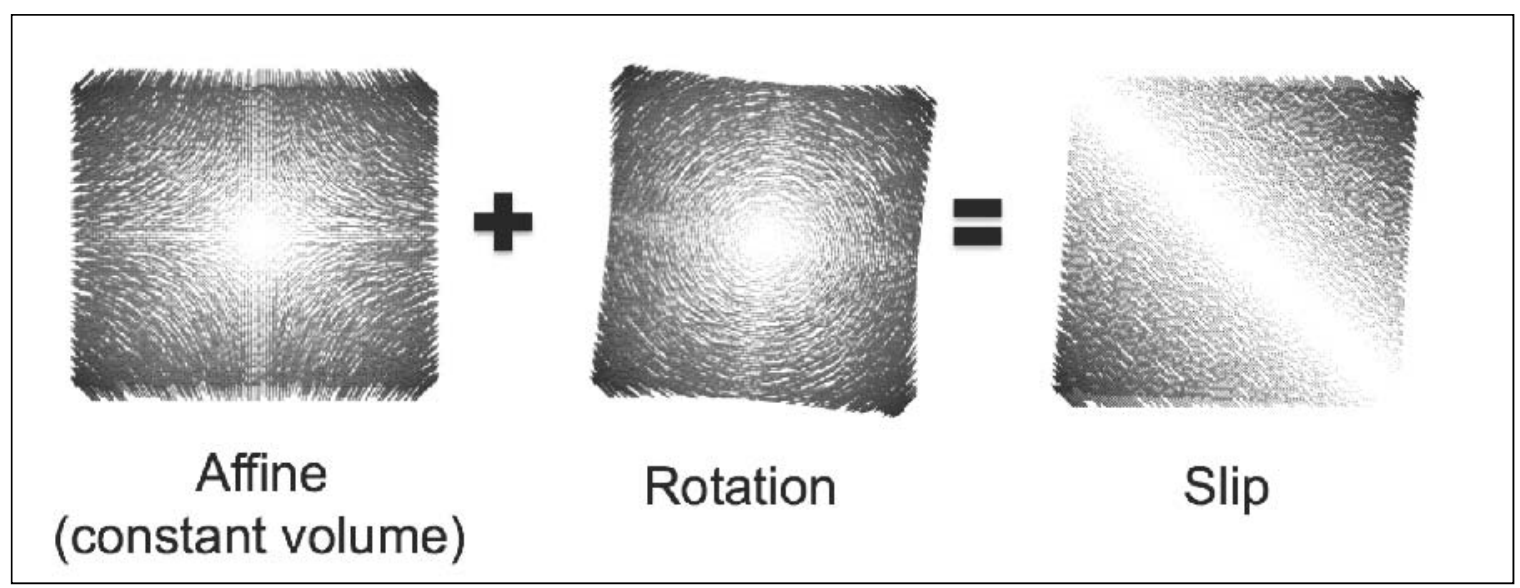

Figure 7. A slip field created from the addition of a uniform strain and a rotation.

where the number of swirls created in the $x_{1}$ and $x_{2}$ directions are dictated by $m$ and $n$, respecitvely (Figure 6). For the case of $m=2$ and $n=1$, the two-swirl case is obtained (Figure 8) which, when added to the affine field, yields a total flow with two shear bands meeting at the boundary similar to those observed in Figure 2.

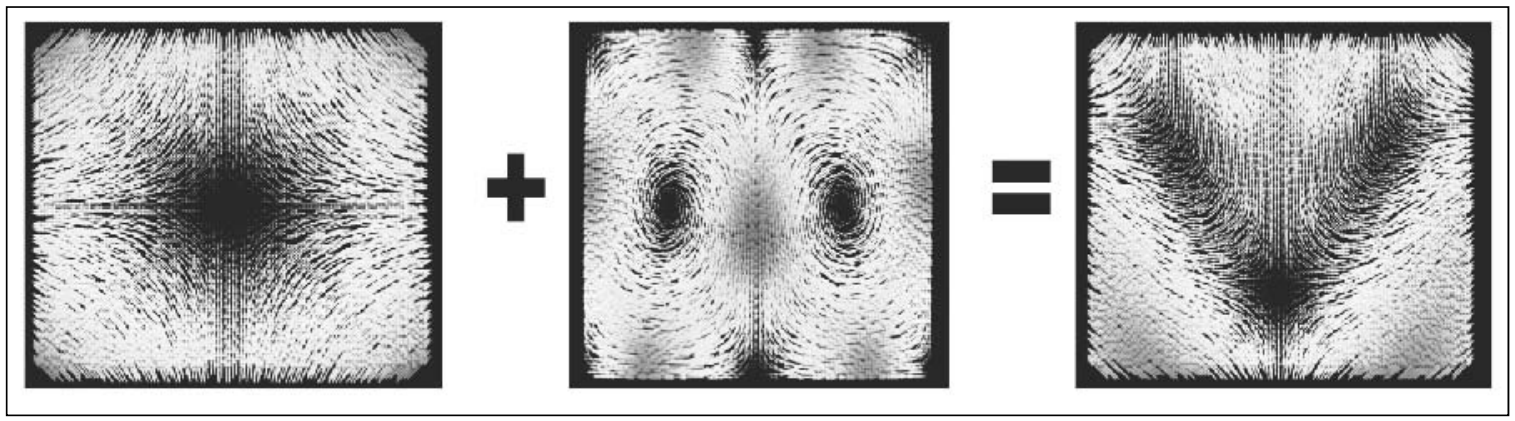

Figure 8. Creation of a double shear banding pattern from addition of uniform strain to rotation field with mirror symmetry.

\subsubsection{Particle rotations}

Particle rotations are degrees of freedom that are independent from the rotation of the particulate medium. For the large-scale deformation, the rotational degrees of freedom consist of an average particle rotation and the gradient of rotation (Appendix A). When the deformation is uniform, both these degrees of freedom are zero, although both particle rotations and their gradients might be significant at the sub-scales. The structure of these subscale rotations is an important consideration. Does the swirl pattern, which is globally rotational, control the rotation of the individual particles? If so, considerable simplification can be imparted to the model because the particle rotation would not be independent of the sub-scale linear particle 
motion. Instead, the particle rotations would behave like the constrained Cosserat medium described by Vardoulakis and Sulem (1995). In that case, the least-square model for determining $\alpha_{i}$ becomes

$$
a=\left(L^{t} L\right)^{-1} L v^{\prime}
$$

where similar to the format described in the appendix,

$$
\begin{aligned}
& v^{\prime}=\left\{\begin{array}{l}
v_{1}^{\prime} \\
v_{2}^{\prime} \\
\omega_{3}^{\prime}
\end{array}\right\} \\
& a=\left\{\begin{array}{l}
\alpha_{1} \\
\alpha_{2}
\end{array}\right\}
\end{aligned}
$$

and

$$
L^{p}=\left[\begin{array}{cc}
F_{1}^{p} & 0 \\
0 & F_{2} \\
F_{2,1}^{p} & -F_{1,2}^{p}
\end{array}\right],
$$

with the functions $F_{i}(x)$ obtained by setting $m=2$ and $n=1$ in Equation 16

$$
F_{1}(x) \sin \left(\frac{\pi x_{1}}{L_{1}}+\pi\right) \cos \left(\frac{\pi x_{2}}{2 L_{2}}+\frac{\pi}{2}\right)
$$

and

$$
F_{2}(x) \cos \left(\frac{\pi x_{1}}{L_{1}}+\pi\right) \sin \left(\frac{\pi x_{2}}{2 L_{2}}+\frac{\pi}{2}\right) .
$$

The spin attributable to the swirl pattern is given by

$$
w_{3}^{\prime}=-\pi\left[\frac{\alpha_{1}}{2 L_{2}}-\frac{\alpha_{2}}{L_{1}}\right] \sin \left(\frac{2 \pi x_{1}}{L_{1}}+\pi\right) \sin \left(\frac{\pi x_{2}}{L_{2}}+\frac{\pi}{2}\right) \text {. }
$$




\subsubsection{Conjugate forces}

The total deformation is depicted by two fields (i.e. an affine field corresponding to a uniform strain $\varepsilon$ and a non-affine field) given in Equation 16 and parameterized by $\alpha_{i}$. It follows that the parameters $\varepsilon$ and $\alpha_{i}$ each should have a conjugate force. These forces can be computed from DEM data by considering the work produced by the virtual increments $\delta \varepsilon$, and $\delta \alpha_{i}$. Internal work is performed solely at particle contacts. For increments $\delta$ ui $(x)$ and $\delta \omega_{i}^{c}$, the work increment is given by

$$
\delta W^{1}=\sum_{c \in N c} f_{i}^{c} \delta u_{i}^{c}+\mu_{i}^{c} \delta w_{i}^{c},
$$

where $f_{i}^{c}$ and $\mu_{i}^{c}$ are the contact force and moment, respectively, with

$$
\delta u_{i}^{c}=\delta u_{i}^{B}-\delta u_{i}^{A}+e_{i j k}\left(R_{j}^{B c} \delta w_{k}^{B}-R_{j}^{A c} \delta w_{k}^{A}\right)
$$

and

$$
\delta w_{i}^{c}=\delta w_{i}^{B}-\delta w_{i}^{A} .
$$

The increments $\delta u_{i}^{A}$ and $\delta u_{i}^{B}$ are the linear motions of the particles making up the contact, and $\delta w_{i}^{A}$ and $\delta w_{i}^{B}$ are the incremental particle rotations. The vectors $R_{j}^{A c}$ and $R_{j}^{B c}$ connect the centers of particle rotation with the contact. The relevant quantities are illustrated in Figure 9.

From a continuum standpoint, the internal work can be expressed in terms of the various components comprising the total velocity field and their conjugates.

$$
\delta W^{I}=\int_{v}\left(\sigma_{i j} \delta \in_{i j}+\alpha_{i} \beta_{i}+\mu_{i}^{o} w_{i}+\mu_{i j} w_{i, j}\right) d V
$$

Note that the rotations $\omega_{i}$ and their gradients $\omega_{i, j}$ must be added to the continuum kinematics as counterparts to the independent particle rotations.

The discrete and continuous kinematic variables for displacement are related through

$$
\delta u i=\delta \in i j x j+\delta \alpha i F i(x) \quad(\text { nosumonrepeated } i) .
$$




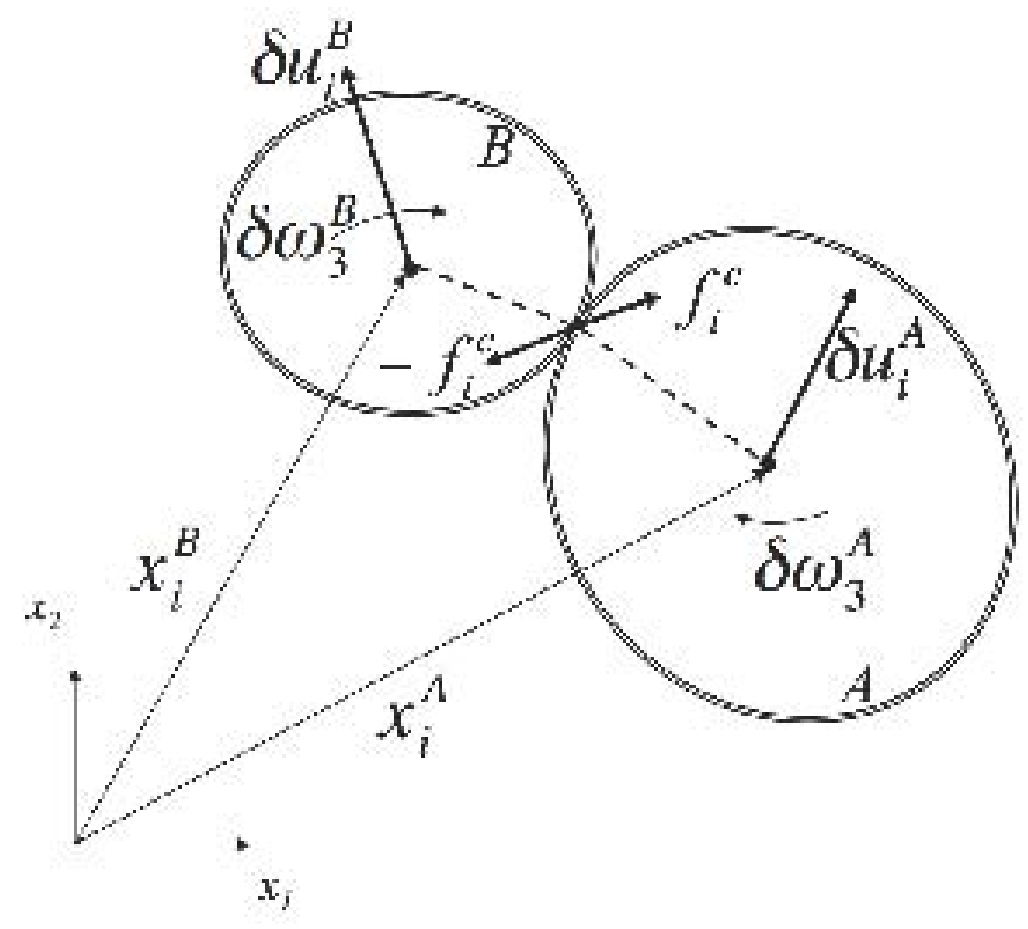

Figure 9. Components comprising the relative contact motion and contact force.

For the rotational variables, the situation is more complex. The globalscale rotational variables $\omega_{i}$ and $\omega_{i, j}$ are zero. As described in sections 2.3.1 and 2.3.2, analysis assumes that the sub-scale rotations are constrained to the spin of the swirl motion. Therefore,

$$
\delta w_{3}=\frac{1}{2}\left(F_{i, j}(x) \delta \alpha_{i}-F_{j, i}(x) \delta \alpha_{j}\right) \text { (nosumon repeatedindex). }
$$

Combining these equations and gathering terms associated with $\delta \in_{i j}$ and $\delta \alpha_{i}$, the following relationships are obtained:

$$
\sigma_{i j}=\frac{1}{V} \sum_{c \in N_{c}} f_{i}^{c}\left(x_{j}^{B}-x_{j}^{A}\right)
$$

and

$$
\beta_{i}=\beta_{i}^{u}+\beta_{i}^{r},
$$

where 


$$
\left.\beta_{i}^{u}=\frac{1}{V} \sum_{c \in N_{c}} f_{i}^{c}\left(F_{i}\left(x^{B}\right)-F_{i}\left(x^{A}\right)\right) \text { (nosumon repeated } i\right)
$$

and

$$
\beta_{i}^{r} \frac{1}{V} \sum_{c \in N_{c}}\left(1-\delta_{i j}\right)\left[f_{i}^{c}\left(R_{2}^{B} F_{i, j}-R_{2}^{A} F_{i, j}^{B}\right)+f_{2}^{c}\left(R_{1}^{B} F_{i, j}-R_{1}^{B} F_{i, j}^{B}\right)\right] .
$$

Equation 24 is the well-known expression for average stress and is exactly equal to the average particle stress given by

$$
\bar{\sigma}_{i j}=\sum_{p \in N_{p}} \frac{1}{V_{p}} \sum_{c \in N_{p c}} f_{i}^{c} x_{j}^{c}
$$

where the subscript $p$ refers to the particle, $c$ to the contact such that $N_{P}$ is the number of particles, $N_{p c}$ is the number of contacts for particle $p$, and $f_{i}^{c}$ is the force acting on the contact located at $x_{j}^{c}$ relative to the particle center. Equation 25 is the conjugate force associated with the swirl magnitudes $\alpha_{i}$.

The force $\beta_{i}$ is a macro-scale quantity because it is conjugate to $\alpha_{i}$, which parameterizes the global swirl pattern. For evaluation of Equation 2, data must be obtained from sub-sampling the domain. 


\section{Results and Discussion}

\subsection{Results}

The least-square fit of the strain increments shown in Figure 10 reflect the constant strain rate imposed by the movement of the platens. However, the plot likewise displays the effects of disruptions to internal motion caused by the kinetic energy bursts. These bursts create fluctuations in the least-square shear strain despite the constant strains imposed.

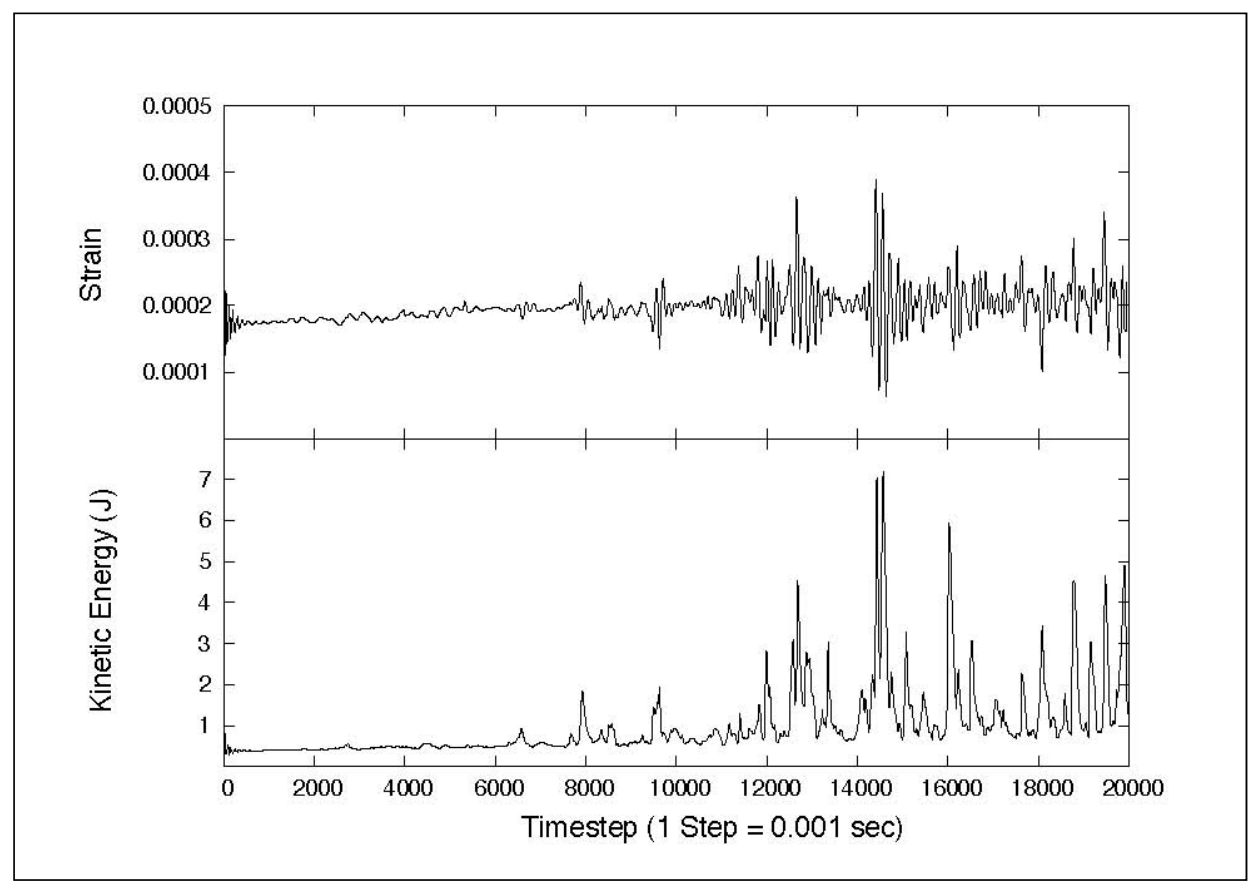

Figure 10. Relationship between strains computed for least-square fit of particle motions and total kinetic energy of particles.

The least-square approximation to the swirl parameters, $\alpha_{i}$, is shown in Figure 11. Similar to the plot for strain in Figure 10, the rate of swirl motion responds to kinetic energy bursts but is markedly different by its continual rise as loading proceeds. Over the course of loading, the magnitude of one of the components of the swirl rate nearly doubles (from about-0.05 to -0.1). The forces conjugate to the swirl motion shown in Figure 12 likewise are similar to the average stress shown in Figure 3. In both cases, the initial loads rise smoothly but become more erratic in response to kinetic energy bursts. The rate of bursts increases as the load plateaus such that the load 


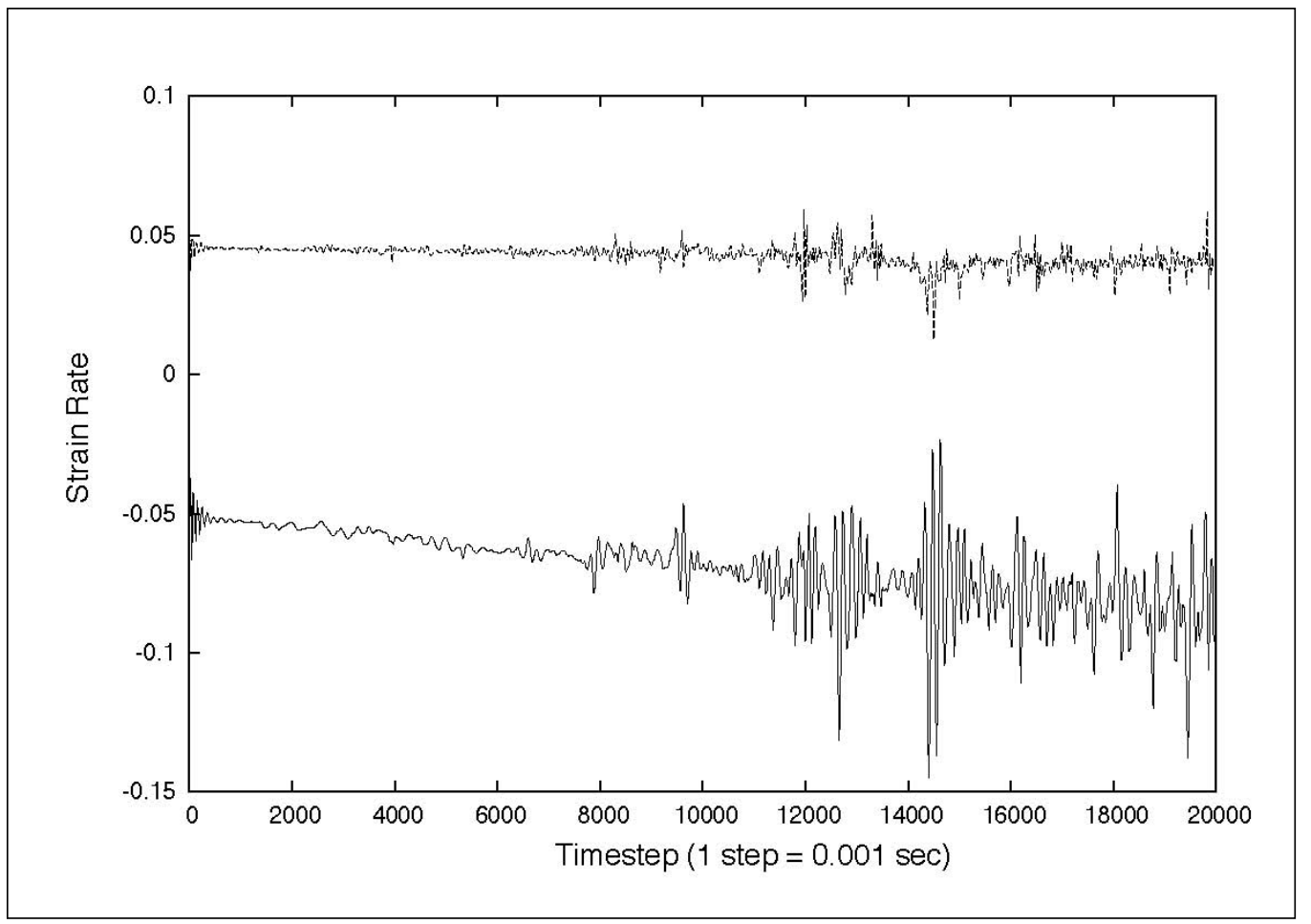

Figure 11. Rate of swirl deformation based on least-square fit showing the early development of the swirl motion.

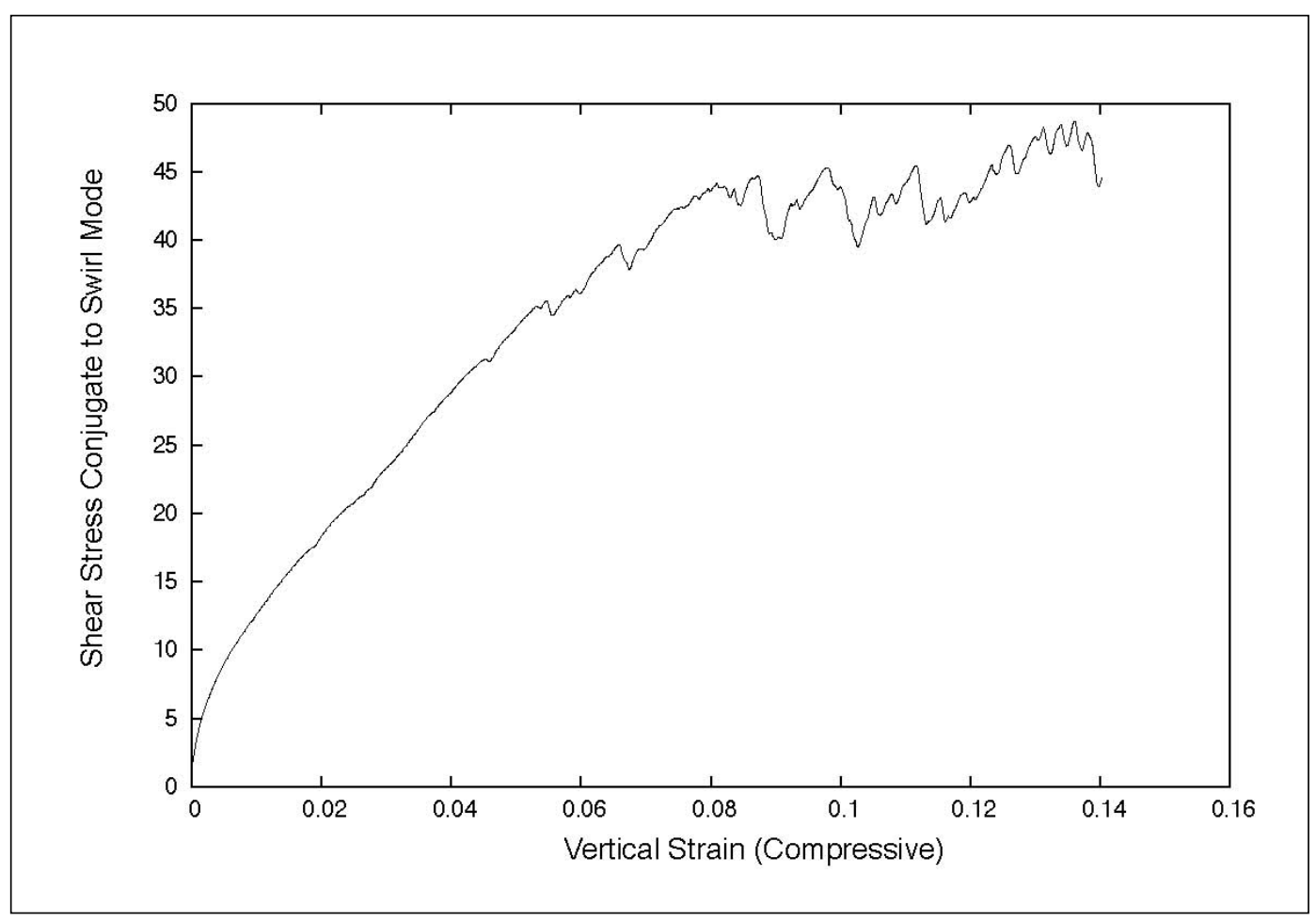

Figure 12. Development of force conjugate to the swirl deformation mode. 
fluctuates about the mean limiting stress. This sequence is explained in detail by Tordesillas et al. (2008), who proposed that the response consists of definable episodes. In the final episode, the pattern of bursts continues but with a distinct reduction on the mean load as the specimen enters its softening phase. It is in this final phase that the swirl patterns thin to a localized shear band.

Particle rotation magnitudes are greatest in regions that correspond roughly to the swirl location. Figure 13 shows the continuum spin computed from Equation 17 at each particle location, where a correspondence between rotations of the particles and the spin at particle locations is apparent from comparisons of the red and blue particles. To confirm this apparent relation, the theoretical spin and the actual particle rotations are compared in a scatter plot, as shown in Figure 14. Ideally, the ranges of both the theoretical and actual spins should be identical; however, the plot shows a clearly discernible trend. This trend suggests that the swirl modes do explain much of the variation in particle rotation, although local deviation from the global trend is evident.

An important observation that was apparent from videos created from the simulations was the tendency for the particles to move in relatively rigid groups, much like chunks of ice in a floe. The particles in these groups rotated at a rate of the rigid-body rotation of the group. The actual deformation occurred between groups.

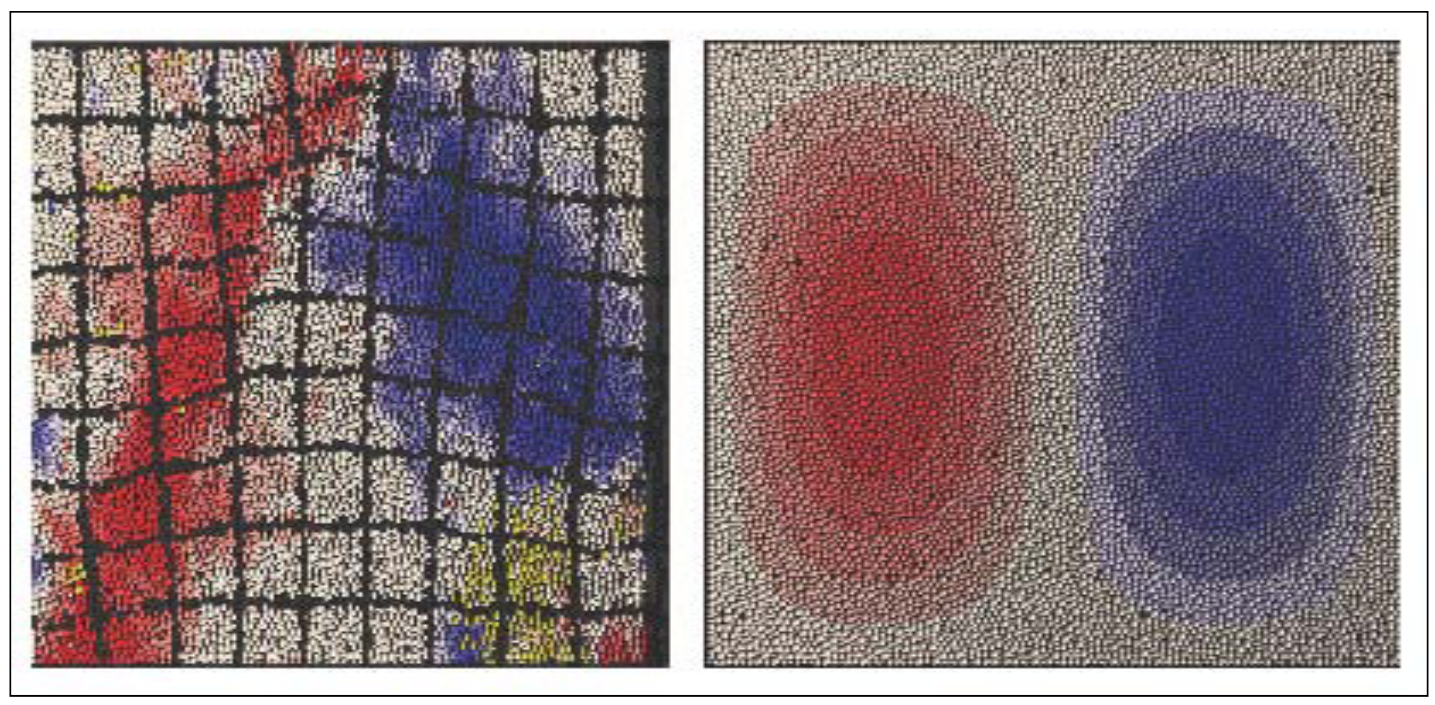

Figure 13. Particle rotations after full pattern development (left) and computed spin (right). 


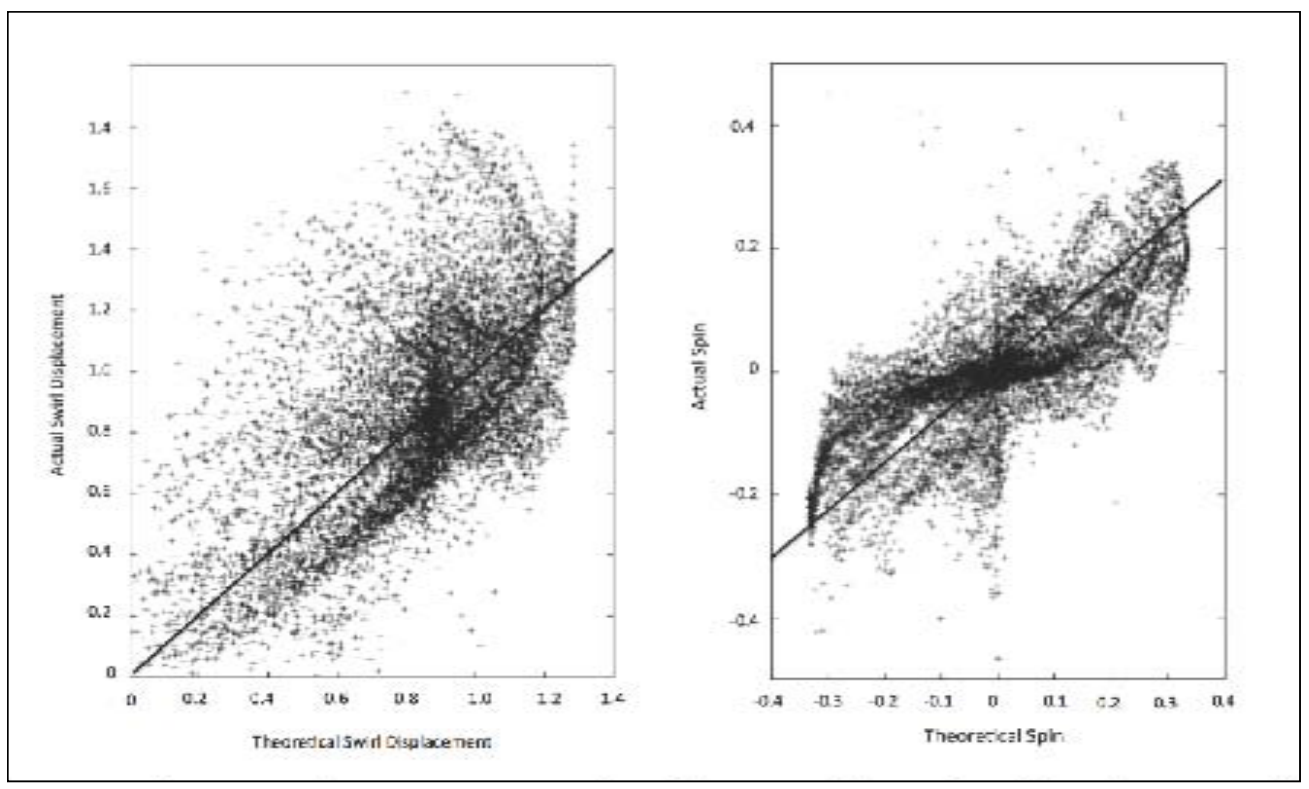

Figure 14. A scatterplot comparing actual and theoretically predicted displacements (left) and spins (right) for each particle in the swirl field.

\subsection{Discussion}

The swirl patterns bring up three issues. The pressing question is what drives the swirl motion, since that is central to defining the coupling between the uniform strain field and the swirl field. Associated with the driving force issue is the nature of the symmetries seen in the swirl pattern that to some extent can be explained by the boundary conditions, yet are not uniquely defined by them. Finally, the relationship between the swirl pattern and the resulting shear band can be used as a visualization tool to explore the full evolution of the shear band, shedding new light on the role of boundary conditions, which conventionally are not considered in shear banding theory, and instability.

\subsubsection{Driving force}

The multi-scale approach is based on treating the non-affine deformation as a second field, with its own set of governing equations. Denoting the non-affine motion as $q_{i}$ and its conjugate force $Q_{i}$, the boundary conditions for the non-affine field are:

$$
q_{i} n_{i}^{w}=0
$$

and 


$$
Q_{i} m_{i}^{w}=0
$$

where $n_{i}^{w}$ is the unit vector normal to wall $w$, and $m_{i}^{w}$ is unit vector tangential to wall $w$, with $n_{i}^{w} m_{i}^{w}=0$. The first condition results from particles touching the wall moving with the wall, making the normal component of the non-affine deformation zero at the wall, as is readily verified by inspection of Figure 6. The second condition is the result of the walls being frictionless, which makes all tangential forces zero. Thus, the boundary conditions for the non-affine field are null. However, the internal forces conjugate to the non-affine motions are not zero, and internal work is performed. If the swirl motion is viewed as a second solution to the boundary value problem representing the biaxial experiment, it must spring from a quiescent state implying that the motion arises from instabilities in the constitutive equations for the non-affine field or from a coupling between the affine and non-affine fields. Focusing on the second possibility, consider that heterogeneity causes the particles to have a diffusive-like random motion leading to patterned velocity fields very much like we see in fluids. In the case of fluids, such motions arise from instabilities caused by variations in buoyancy forces, which are absent in the biaxial test because of the absence of gravity. Thus, although random motions caused by heterogeneity make the particles mobile, the driving forces to create organized patterns are missing. The coupling between affine and non-affine fields is suggested by the manner that both motions and forces follow in lock-step with the affine fields. One possibility is that force-chain buckling, which by definition creates non-affine motion, is an energy transfer mechanism between the two fields. In such a mechanism, applied forces at the boundaries impart strain energy at contacts of those particles, making up force chains, causing jamming. As force chains break down, energy is released as kinetic energy (Figure 3), which is available to drive particle diffusion. In this scenario, the swirl pattern results from the boundary conditions by which particles must move tangential to the walls. To improve on this admittedly vague description, the dependence of the free energy, psi, in Equation 2 on affine and non-affine deformations must be defined, which is beyond the limited scope of this study. The coupling between the two deformation fields is defined by the free energy relationship. The lack of analysis notwithstanding, it is useful to consider the sources of regularity in the motions and in the implications on shear band patterns. 


\subsubsection{Symmetry of swirls}

It might seem reasonable that there should be symmetrical patterns because the loading and geometry have symmetry. For the case of frictionless boundaries, there are two swirl patterns whereas, by adding friction to the walls, four swirls are created (Figure 6). Yet, Equation 16 permits an unlimited number of possible swirl patterns.

Even though we think of the problem as pseudo-static, it is actually dynamic. The top and bottom boundaries are advancing into the particles, and the side boundaries are moving from the particles. Thus, there are two sets of waves propagating from the boundaries moving toward the center. The sets from the top and bottom are increasing the compressive forces; the sets from the sides are reducing the compressive particle forces. Therefore, even if the material is homogeneous, the stress state is not. There is a twofold symmetry in the stress state. If we rotate the specimen $180 \mathrm{deg}$, we get the same picture back.

The issue of symmetry is made interesting because of the rotational pattern. Notice that there must be a mirror symmetry in the rotations when there is an even number of swirls. There is a right-hand and lefthand version of each swirl pattern, depending on the signs of $\alpha i$. The choice of which must be happenstance because there is no way to distinguish up or down in these simulations (i.e. no gravity).

So why does the case of frictionless sides give rise to the double-swirl pattern? Heterogeneity causes motion in the form of particle migration, but the migration is constrained by the boundary conditions to be rotational. The symmetry of the system is such that only certain patterns will emerge, each with a mirror-like symmetry. Finally, with regard to why the swirls appeared in pairs, one can conjecture that the two-swirl case dissipates less energy and therefore is preferred. Thus, the number of swirls that is formed will be the number dissipating the least energy while fitting the symmetry of the boundary conditions.

\subsubsection{Bifurcation}

The development of patterned deformations that lead to localization begs the question of how these patterns are related to bifurcations in the solution of the boundary value problem. In the case of the biaxial test, from a continuum point of view, one solution corresponds to constant 
homogeneous strain (i.e. the so-called trivial solution). The bifurcation point comes when a second solution appears consisting of sliding motion that usually ends as a localized zone of shear. Noting the previously discussed relationship between the swirl pattern and the shear band, the emergence of the shear band is not an abrupt event. Swirl patterns seem to emerge from the start of loading and grow in intensity as the loading proceeds. It is as if they are a background motion associated with the jamming-kinetic energy burst sequence. Each time there is a burst of kinetic energy, the pattern becomes better defined. The existence of precursor events to failure in biaxial simulations has been documented by Welker and McNamara (2011).

It is reasonable that the shear banding that is seen is the result of growth of non-affine motion that satisfies its own set of evolution equations. By this approach, there is not really a bifurcation from one solution but rather a competition between two or more (possibly coupled) solutions, each with its own boundary conditions and each present throughout the history of loading. More important, it is not necessary to define a condition for the onset of localization. Instead, the banding evolves naturally as a result of the combined deformation patterns. The resistance to shear localization is the forces necessary to drive the swirl pattern $\left(\beta_{i}\right)$ and is a measurable quantity using the results from the simulated experiment.

Although the swirl patterns appear to define the locations of the shear bands, additional processes must be involved to create the final localized band. The swirl pattern initiates the rotational motion in the region where deformation localizes. Once this motion becomes significant, force-chain buckling leads to stress reduction (softening) within the band region. Thinning of the band is the result of the bifurcated path in the unload behavior of the material, whereby the material either can unload elastically or continue loading plastically with a declining stress due to softening. Both paths correspond to stress reduction, thus the elastic unloading and the plastic softening region can coexist while maintaining static equilibrium. The boundary between the two regions can move, but only to increase the size of the elastic region; once a region has unloaded elastically, it falls below the yield limit and will not re-plasticize unless the boundary conditions are changed. See Tordesillas et al. (2004) for further discussion on this shear zone thinning mechanism. 


\section{Conclusions}

Non-uniform motions emerge in loading configurations, such as biaxial loading, that should display uniform strain. These motions are a result of inhomogeneity of the discrete media and instabilities in the inter-particle interactions. These motions are revealed by subtracting the projected motions due to uniform strain from the total observed motion. Thus, the total particle motion consists of an affine part, consistent with uniform strain, and a non-affine part. The non-affine motion in the biaxial test, denoted as swirls, is a rotational motion that satisfies the kinematic boundary condition at the particle-platen boundary. Particularly revealing is the observation that sliding motion can be represented as the sum of affine motion and rotation, which suggests an avenue to develop an evolution relationship for localization. Central to such a relationship is defining the conjugate force associated with the swirl motion. To that end, the swirl motion was parameterized through a Fourier representation that associated constants with each swirl mode. Through application of virtual work arguments, forces were associated with each swirl mode, allowing the complete evolution of motion and force to be determined. The swirl motion and its conjugate force grow continuously from the outset of loading. The association between the swirl pattern and localization implies that shear banding in the biaxial test is not a sudden bifurcation, but is the end result of a continuous process that is influenced by boundary conditions as well as internal instabilities. This work lays the foundation for a multi-scale analysis of granular media within a continuum context. 


\section{References}

Bagi, K. 2006. Analysis of microstructural strain tensors for granular assemblies. International Journal of Solids and Structures 43(10):3166-3184.

Bardet, J . P., and J . Proubet. 1992. Shear-band analysis in idealized granular material. Journal of Engineering Mechanics 118(2):397-415.

Bazant, Z. P., and T. B. Belytschko. 1985. Wave propagation in a strain-softening bar: Exact solution. Journal of Engineering Mechanics 111(3):381-389.

Cosserat, E., and F. Cosserat. 1909. Théorie des Corps déformables. Herman et fils, Paris.

Cundall, P. A., and O. D. L. Strack. 1979. A discrete numerical model for granular assemblies. Geotechnique 29(1):47-65.

De Borst, R., and L. J . Sluys. 1991. Localisation in a cosserat continuum under static and dynamic loading conditions. Computer Methods in Applied Mechanics and Engineering 90(1-3):805-827.

Gaspar, N., and M. A. Koenders. 2001. Micromechanic formulation of macroscopic structures in a granular medium. Journal of Engineering Mechanics 127:987.

Germain, P. 1973. The method of virtual power in continuum mechanics, part 2: Microstructure. SIAM Journal on Applied Mathematics 556-575.

Horner, D. A., J . F. Peters, and A. Carrillo. 2001. Large scale discrete element modeling of vehicle soil interaction. Journal of Engineering Mechanics 127(10):1027-1032.

Kadowaki, H., and W. K. Liu. 2004. Bridging multi-scale method for localization problems. Computer Methods in Applied Mechanics and Engineering 193(3032):3267-3302.

Kadowaki, H., and W. K. Liu. 2005. A multiscale approach for the micropolar continuum model. CMES: Computer Modeling in Engineering \& Sciences 7(3):269-282.

Koenders, M. A. 1994. Least squares methods for the mechanics of nonhomogeneous granular assemblies. Acta Mechanica 106(1):23-40.

Kuhn, M. R. 1999. Structured deformation in granular materials. Mechanics of Materials 31(6):407-429.

Kuhn, M. R. 2003. Heterogeneity and patterning in the quasi-static behavior of granular materials. Granular Matter 4(4):155-166.

Lade, P. V., P. A. Bopp, and J . F. Peters. 1993. Instability of dilating sand. Mechanics of Materials 16(3):249-264.

Liu, W. K., E. G. Karpov, and H. S. Park. 2006. Nano mechanics and materials: Theory, multiscale methods and applications. J ohn Wiley \& Sons. 
Matous, K., H. M. Inglis, X. Gu, D. Rypl, T. L. J ackson, and P. H. Geubelle. 2007. Multiscale modeling of solid propellants: From particle packing to failure. Composites Science and Technology 67(7-8):1694-1708.

McDowell, D. L. 2000. Modeling and experiments in plasticity. International Journal of Solids and Structures 37(1-2):293-309.

Mindlin, R. D. 1964. Micro-structure in linear elasticity. Archive for Rational Mechanics and Analysis 16(1):51-78.

Mogami, T., and K. Kubo. The behavior of soil during vibration. In Proceedings of the Third International Conference of Soil Mechanics and Foundation Engineering, 1:152-155. Reprinted in Selected Papers of Takeo Mogami, Gihodo Publishing Co., 1974, 1953.

Mühlhaus, H. B., and I. Vardoulakis. 1987. The thickness of shear bands in granular materials. Géotechnique, 37(3):271-283.

Peters, J . F., P. V. Lade, and A. Bro. 1986. Advanced triaxial testing of soil and rock, chapter shear band formation in triaxial and plane strain tests. ASTM STP 977, pages 604-627.

Peters, J . F. 2005. Some fundamental aspects of the continuumization problem in granular media. Journal of Engineering Mathematics 52(1):231-250.

Radjai, F., and S. Roux. 2002. Turbulentlike fluctuations in quasistatic flow of granular media. Physical Review Letters 89(6):64302.

Rechenmacher, A. L. 2006. Grain-scale processes governing shear band initiation and evolution in sands. Journal of the Mechanics and Physics of Solids 54(1):22-45.

Rice, J . R. 1977. The localization of plastic deformation. In Proceedings of the 14th IUTAM Congress, 1:207. North Holland.

Rudnicki, J. W., and J.R. Rice. Conditions for the localization of deformation in pressuresensitive dilatant materials. Journal of the Mechanics and Physics of Solids 23(6):371- 394, 1975.

Sluys, L. J . 1992. Wave propagation, localisation and dispersion in softening solids. Ph.D thesis, Proefshrift Technische Universiteit, Delft.

Tordesillas, A., and D. C. Walsh. 2002. Incorporating rolling resistance and contact anisotropy in micromechanical models of granular media. Powder Technology 124(1-2):106-111.

Tordesillas, A., J . F. Peters, and B. S. Gardiner. Shear band evolution and accumulated microstructural development in Cosserat media. International Journal for Numerical and Analytical Methods in Geomechanics 28(10):981-1010, 2004.

Tordesillas, A., M. Muthuswamy, and S. D. C. Walsh. 2008. Mesoscale measures of nonaffine deformation in dense granular assemblies. Journal of Engineering Mechanics 134(12):1095-1113. 
Tordesillas, A., Q. Lin, J . Zhang, R. P. Behringer, and J . Shi. 2010. Structural stability and jamming of self-organized cluster conformations in dense granular materials. Journal of the Mechanics and Physics of Solids 59:265-296.

Utter, B., and R. P. Behringer. 2009. Multiscale motion in the shear band of granular couette flow. In Proceedings, AIP Conference 1145:339-342.

Valanis, K. C. 1996. A gradient theory of internal variables. Acta Mechanica 116(1):1-14, 1996.

Valanis, K. C. A gradient thermodynamic theory of self-organization. Acta Mechanica 127(1):1-23, 1998.

Valanis, K. C. 2001. Quantum plasticity. In Bifurcation and localisation theory in geomechanics: Proceedings of the 5th International Workshop on Bifurcation and Localisation Theory in Geomechanics, Perth, 29 November-2 December 1999. eds. H. B. Mühlhaus, A. Dyskin, and E. Pasternak, 103. Taylor \& Francis.

Valanis, K. C., and J . F. Peters. Ill-posedness of the initial and boundary value problems in non-associative plasticity. Acta mechanica 114(1):1-25, 1996.

Vardoulakis, I., and E. C. Aifantis. A gradient flow theory of plasticity for granular materials. Acta Mechanica 87(3):197-217, 1991.

Vardoulakis, I., and J. Sulem. 1995. Bifurcation analysis in geomechanics. Blackie Academic \& Professional Publishers, Glasgow.

Walsh, S. D. C., and A. Tordesillas. 2006. Finite element methods for micropolar models of granular materials. Applied Mathematical Modelling 30(10):1043-1055.

Welker, P., and S. McNamara. 2011. Precursors of failure and weakening in a biaxial test. In Granular Matter, 1-13, ISSN 1434-5021.

Williams, J. R., and N. Rege. 1997. Coherent vortex structures in deforming granular materials. Mechanics of Cohesive-frictional Materials 2(3):223-236. 


\section{Appendix A: Data Structures}

For the projection

$$
u=L e,
$$

the strain from the uniform continuum motion e that gives an unbiased fit for the collection of particle motions $u$ is found from the relationship

$$
e=\left(L^{T} L\right)^{-1} L u
$$

The motion of a particular particle $p$ is given by

$$
u^{p}=L^{p} e .
$$

Accordingly, $u$ has the structure

$$
u=\left\{\begin{array}{c}
u^{1} \\
u^{2} \\
\vdots \\
u^{N_{p}}
\end{array}\right\}
$$

The matrix $L$ is constructed from $L^{p}$ in a similar way.

The stucture of $L^{p}$ is based on the ordering of terms in $u^{p}$ and $e$. The ordering of $u^{p}$ is as follows:

$$
u^{p}=\left\{\begin{array}{l}
u_{x} \\
u_{y} \\
\omega_{z}
\end{array}\right\} .
$$

The continuum motion $e$ includes the rigid body motion $u_{i}^{0}$; uniform strain $\epsilon_{i j}$; independent particle rotation (Cosserat rotation) $w_{i}$; and rotation gradient $K_{i j}$. 


$$
e^{T}=\left\{v_{x}^{o}, v_{y}^{o}, w_{z}^{o}, \in_{x x}, \in_{y y}, \in_{x y}, W_{z}, K_{z x}, K_{z y},\right\}
$$

The vector $e$ can be expressed in terms of sub-groups given by

$$
e T=\left\{u^{o}, w_{z}^{o}, e^{s}, w_{z}, k\right\}
$$

Similarly, $u$ can be grouped as

$$
u^{p}=\left\{u, w_{3}\right\}^{p}
$$

The matrix $L^{p}$ now can be expressed in matrix/tablar form

\begin{tabular}{c|ccccc} 
& $u^{o}$ & $w_{z}^{o}$ & $e^{s}$ & $w_{z}$ & $k$ \\
\hline$u^{p}$ & $I$ & $R$ & $x$ & $o$ & $o$ \\
$w_{3}^{p}$ & $o$ & 1 & $o$ & 1 & $x^{k}$
\end{tabular},

where $I$ is a $2 \times 2$ identity matrix, 0 matrices of zeros of appropriate dimension, and

$$
\begin{gathered}
R=\left[\begin{array}{c}
y \\
-x
\end{array}\right], \\
x=\left[\begin{array}{lll}
x & 0 & y \\
0 & y & x
\end{array}\right], \\
x k=\left[\begin{array}{ll}
x & y
\end{array}\right] .
\end{gathered}
$$




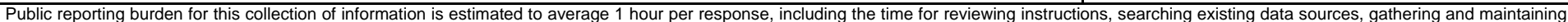

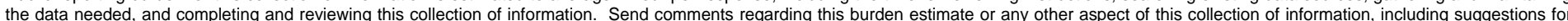

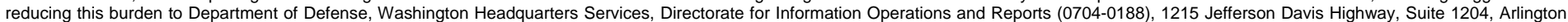

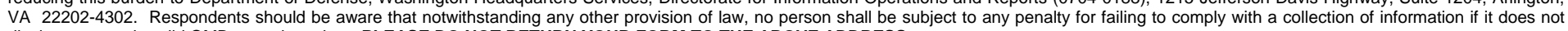
display a currently valid OMB control number. PLEASE DO NOT RETURN YOUR FORM TO THE ABOVE ADDRESS.
1. REPORT DATE (DD-MM-YYYY) 2. REPORT TYPE
3. DATES COVERED (From - To)

August 2012 Final

\section{TITLE AND SUBTITLE}

Military Engineering: Patterned Non-affine Motion in Granular Media

5a. CONTRACT NUMBER

5b. GRANT NUMBER

5c. PROGRAM ELEMENT NUMBER

\section{AUTHOR(S)}

John F. Peters and Laura E. Walizer

5e. TASK NUMBER

5f. WORK UNIT NUMBER

\section{PERFORMING ORGANIZATION NAME(S) AND ADDRESS(ES)}

8. PERFORMING ORGANIZATION REPORT

Geotechnical and Structures Laboratory

U.S. Army Engineer Research and Development Center NUMBER

3909 Halls Ferry Road

ERDC/GSL TR-12-28

Vicksburg, MS 39180-6199

\section{SPONSORING I MONITORING AGENCY NAME(S) AND ADDRESS(ES)}

U.S. Army Corps of Engineers, Washington, DC 20314-1000

10. SPONSOR/MONITOR'S ACRONYM(S)

U.S. Army Engineer Research and Development Center Military Engineering Basic Research Program, Fundamental Investigation on Bridging Between Microsale Phenomena and Macrosale Performance

12. DISTRIBUTION I AVAILABILITY STATEMENT

Approved for public release; distribution is unlimited.

\section{SUPPLEMENTARY NOTES}

\section{ABSTRACT}

Vortex-like flow patterns often are observed in experiments on granular media for which uniform strain is expected based on the loading boundary conditions. These deformations become apparent when the motion associated with uniform strain is subtracted from the total particle motion. Besides presenting an interesting phenomenon that begs explanation, these vortex patterns suggest multi-scale structure to non-affine motion as suggested by modern continuum theories. Further, the rotational velocity field added to a uniform strain field produces a planar slip field. Thus, these structures are associated with the slip-band fields that eventually form and generally are associated with bifurcations in the solution path of the governing partial differential equations. A procedure is presented to extract these motion fields from discrete element simulations, along with conjugate forces associated with these motions. A key finding from the simulations is that the motions that eventually lead to shear band formation develop throughout the loading history rather than arising as a distinct bifurcation. Further, the pattern of rotational fields and, hence, the shear banding pattern are controlled by the boundary conditions. A question, only partly resolved here, is the origin of forces driving the rotational fields, considering no force exists at the boundaries in the case of frictionless platens.

\begin{tabular}{lll}
\hline 15. SUBJECT TERMS & Non-local continuum & Localization \\
Affine motion & Hierarchal & Granular media
\end{tabular}

Multi-scale Discrete Element Method (DEM)

16. SECURITY CLASSIFICATION OF:

17. LIMITATION

a. REPORT OF ABSTRACT

\begin{tabular}{|c|} 
18. NUMBER \\
OF PAGES \\
\cline { 2 - 2 }
\end{tabular}

19a. NAME OF RESPONSIBLE PERSON

19b. TELEPHONE NUMBER (include

Unclassified

b. ABSTRACT
Unclassified

c. THIS PAGE

Unclassified area code) 